Series A

\title{
Revenue maximization in Stackelberg Pricing Games: beyond the combinatorial setting
}

\author{
Toni Böhnlein ${ }^{1} \cdot$ Stefan Kratsch ${ }^{2}$. Oliver Schaudt ${ }^{3}$
}

Received: 5 October 2017 / Accepted: 13 March 2020 / Published online: 18 April 2020

(c) The Author(s) 2020

\begin{abstract}
In a Stackelberg Pricing Game a distinguished player, the leader, chooses prices for a set of items, and the other players, the followers, each seek to buy a minimum cost feasible subset of the items. The goal of the leader is to maximize her revenue, which is determined by the sold items and their prices. Most previously studied cases of such games can be captured by a combinatorial model where we have a base set of items, some with fixed prices, some priceable, and constraints on the subsets that are feasible for each follower. In this combinatorial setting, Briest et al. and Balcan et al. independently showed that the maximum revenue can be approximated to a factor of $H_{k} \sim \log k$, where $k$ is the number of priceable items. Our results are twofold. First, we strongly generalize the model by letting the follower minimize any continuous function plus a linear term over any compact subset of $\mathbb{R}_{\geq 0}^{n}$; the coefficients (or prices) in the linear term are chosen by the leader and determine her revenue. In particular, this includes the fundamental case of linear programs. We give a tight lower bound on the revenue of the leader, generalizing the results of Briest et al. and Balcan et al. Besides, we prove that it is strongly NP-hard to decide whether the optimum revenue exceeds the lower bound by an arbitrarily small factor. Second, we study the parameterized complexity of computing the optimal revenue with respect to the number $k$ of priceable items. In the combinatorial setting, given an efficient algorithm for optimal follower solutions, the maximum revenue can be found by enumerating the $2^{k}$ subsets of priceable items and computing optimal prices via a result of Briest et al., giving time $O\left(2^{k}|I|^{c}\right)$ where $|I|$ is the input size. Our main result here is a W[1]hardness proof for the case where the followers minimize a linear program, ruling out running time $f(k)|I|^{c}$ unless FPT $=\mathrm{W}[1]$ and ruling out time $|I|^{o(k)}$ under the Exponential-Time Hypothesis.
\end{abstract}

Keywords Algorithmic pricing · Stackelberg games · Approximation algorithms · Revenue maximization · Parameterized complexity

A preliminary version of this paper will appeared in the Proceedings of the 44th International Colloquium on Automata, Languages, and Programming (ICALP 2017).

Extended author information available on the last page of the article 
Mathematics Subject Classification 68Q17 · 90C47 · 91A46

\section{Introduction}

Pricing problems are fundamental in both economics and mathematical optimization. In this paper we study such pricing problems formulated as games, which are usually called Stackelberg Pricing Games [23]. In our setting, in order to maximize her revenue one player chooses prices for a number of items and one or several other players are interested in buying these items. Following the standard terminology, the player to choose the prices is called the leader while the other players are called followers. Depending on the follower's preferences, computing optimal prices can be a computational non-trivial problem. In a setting where followers have valuations over individual items only, the problem is simple. If, however, valuations become more complex, e.g., over whole subsets of items, pricing problems become much harder-also in a formal sense.

Largely the literature has focused on what we call the combinatorial setting: there is a set $Y$ of items and one follower seeks to buy a feasible subset. Some of the items have fixed costs, the others have prices that are chosen by the leader. If the follower buys a feasible subset $S \subseteq Y$ of the items, he has to pay the sum of the fixed costs of the elements of $S$, plus the leader's prices of the bought elements. The leader's revenue is the sum of the prices of the priceable items in $S$. This can also be captured by defining a solution space $X$ containing 0/1-vectors corresponding to the feasible subsets $S$ of $Y$. The goal of the follower is then to minimize a given additive function $f: X \rightarrow \mathbb{R}$ that depends on both fixed and leader-chosen prices.

So-called Stackelberg Network Pricing Games became popular when Labbé et al. [20] used them to model road toll setting problems. In this game, the leader chooses prices for a subset of priceable edges in a network graph while the remaining edges have fixed costs. Each follower has a pair of vertices $(s, t)$ and wants to buy a minimum cost path from $s$ to $t$, taking into account both the fixed costs and the prices chosen by the leader. The work of Labbé et al. led to a series of studies of the Stackelberg Shortest Path Game. Roche et al. [21] showed that the problem is NP-hard, even if there is only one follower, and it has later been shown to be APX-hard $[8,19]$. More recently, other combinatorial optimization problems were studied in their Stackelberg pricing version. For example, Cardinal et al. [12,13] studied the Stackelberg Minimum Spanning Tree Game, proving APX-hardness and giving some approximation results. Moreover, a special case of the Stackelberg Vertex Cover Game in bipartite graphs has been shown to be polynomially solvable by Briest et al. [10].

To get more familiar with the setting, we briefly discuss an example of the Stackelberg Minimum Spanning Tree Game. The left hand side of Fig. 1 depicts an instance of the problem. Here the leader can choose the prices $p_{1}, p_{2}$ and $p_{3}$ for the dashed edges, while the solid edges have fixed costs as displayed. To motivate the problem, think of the vertices as hubs in a network and of the edges as data connections. In this scenario, the followers are Internet Service Providers and want to connect all the hubs at minimum cost, thus want to compute a minimum spanning tree. The leader owns the 

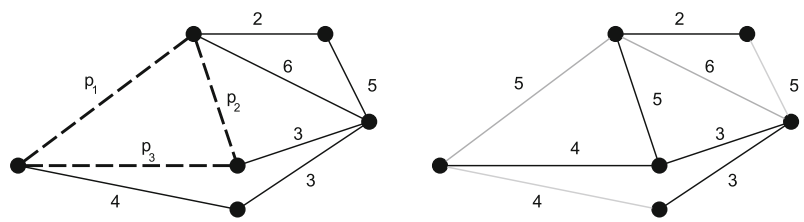

Fig. 1 An instance of the Stackelberg minimum spanning tree game

dashed connections and wants to set prices, that yield a large revenue. Furthermore, there are competitors who own the solid connections and it is known how much they charge for their usage.

On the right hand side, an optimal pricing $\left(p_{1}=p_{2}=5\right.$ and $\left.p_{3}=4\right)$ and a corresponding minimum spanning tree are depicted. Thus the leader's revenue amounts to 9 , which can be verified to be maximum. Observe that we can compute a minimum spanning tree without any priceable edges; otherwise the leader's revenue is unbounded. In this example, the total cost of such a minimum spanning tree is 17 . In contrast, if we set all prices to 0 and let the follower compute a minimum spanning tree, it has a total cost of 8 . The difference of these two values, $17-8=9$, is an upper bound on the revenue of the leader, as explained later. This upper bound, which we denote by $R$, is sometimes called the optimal social welfare and will be important for our approximation result.

An important contribution to the study of Stackelberg Games was the discovery by Briest et al. [10]. They show that the optimal revenue can be approximated surprisingly well using a single-price strategy. For a single-price strategy the leader sets the same price for all of her priceable items. Basically, their result says the following: In any Network Pricing Game with $k$ priceable items, there is some $\lambda \in \mathbb{R}_{\geq 0}$ such that, when assigning the price of $\lambda$ to all priceable items at once, the obtained revenue is only a factor of $H_{k}$ away from the optimal revenue. Here, $H_{k}=\sum_{i=1}^{k} 1 / i$ denotes the $k$ - $t h$ harmonic number which behaves like $\log (k)$. Hence, the maximum revenue can be approximated to a factor logarithmic in $k$.

This discovery has been made independently, in a slightly different model, by Balcan et al. [3]. Actually, in both papers $[3,10]$ a stronger fact is proven: The singleprice strategy yields a revenue that is at least $R / H_{k}$, where $R$ is a natural upper bound on the optimal revenue. The definition of $R$ was sketched in the example above, and is formally laid out later.

Our results Our work focuses on pushing the knowledge on Stackelberg Pricing Games beyond the well-studied combinatorial setting, in order to capture more complex problems of the leader. This is motivated by the simple fact that the combinatorial setting is too limited to even model, e.g., a follower that has a minimum cost flow problema crucial problem in both, combinatorial optimization and algorithmic game theory. More generally, we might want to be able to give bounds and algorithms in the case when the follower has an arbitrary linear or even convex program. For example, the follower might have a production problem in which he needs to buy certain materials from the leader, but such pricing problems haven't been discussed in the literature so far. 
We prove an approximation result that applies even to a setting generalizing linear and convex programs. In our model, the follower minimizes a continuous function $f$ over a compact set of feasible solutions $x \in X \subseteq \mathbb{R}_{\geq 0}^{n}$. For some of the variables, say $x_{1}$ up to $x_{k}$, the leader can choose a price vector $p \in \mathbb{R}^{k}$. Now the follower chooses a vector $x \in X$ that minimizes his objective function $f(x)+\sum_{i=1}^{k} p_{i} x_{i}$. We remark that if $X$ is a set containing 0/1-vectors only, then we are back to the classical combinatorial setting. The result of Briest et al. can be transferred to the case when $f$ is non-additive, in view of their original proof. Moreover if $X$ is a polytope and $f$ is additive, the follower minimizes a linear program, which is an important special case.

In Sect. 2, we formally introduce this more general model and prove the following results.

- The maximum revenue obtainable by the leader can be approximated to a logarithmic factor using a single-price strategy. This generalizes the above mentioned result of Briest et al. [10] not only to linear programs but to any kind of follower that is captured by our model.

- The analysis of point (i) is tight. There is a family of instances for which the single-price strategy yields maximum revenue. And this revenue meets the bound of point (i).

- It is strongly NP-hard to decide whether one can achieve a revenue that is only slightly larger than the one guaranteed by the single-price strategy. This holds true even in a very restricted combinatorial setting.

The second part of the paper deals with the parameterized complexity of Stackelberg Pricing Games (Sect. 3). To the best of our knowledge, the only result in this direction is an XP-algorithm by Cardinal et al. [13] for the Stackelberg Minimum Spanning Tree Game in graphs of bounded treewidth.

In contrast to structural parameters like the treewidth of the input graph, we consider the complexity of the pricing problem when parameterized by the number of priceable variables (or items in the combinatorial setting). Our main result in this part is a W[1]-hardness proof for the case that the optimization problem of the follower is a linear program, which is arguably one of the most interesting cases that does not fit into the combinatorial setting. This rules out algorithms of running time $f(k)|I|^{c}$ unless FPT $=\mathrm{W}[1]$ for any function $f$ and polynomial $|I|^{c}$ of the input size; it also rules out running time $|I|^{o(k)}$ under the Exponential-Time Hypothesis of Impagliazzo et al. [18]. This intractability result is complemented by a fairly simple FPT-algorithm with running time $O\left(2^{k}|I|^{c}\right)$ for any Stackelberg Game that fits into the combinatorial model, when provided with an efficient algorithm for finding optimal follower solutions. The algorithm enumerates all subsets of priceable items and applies a separation argument of Briest et al. [10] to compute optimal leader prices and revenue.

Related work Most important for our work are the approximation results due to Briest et al. [10] and Balcan et al. [3], which were discussed above.

A larger body of work focuses on specific network problems in their Stackelberg Game version. Briest et al. [10] give a polynomial time algorithm for a special case of the Stackelberg Bipartite Vertex Cover Game. An algorithm with improved running time was later given by Baïou and Barahona [2]. As mentioned, Labbé et al. [20] use the Stackelberg Shortest Path Game to model road toll setting problems. They establish 
NP-hardness and use LP bilevel formulations to solve small instances. A combinatorial approximation algorithm with the same logarithmic approximation guarantee as the single-price strategy was given by Roch et al. [21]. Moreover, a lower bound on the approximability is due to Briest et al. [8]: they show that the Stackelberg Shortest Path Game is NP-hard to approximate within a factor of less than 2. This is an improvement over previous results by Joret [19] showing APX-hardness. Further research on the Stackelberg Shortest Path Game can be found in a survey by van Hoesel [22]. A similar problem, the Stackelberg Shortest Path Tree Game, is studied by Bilo et al. [6]. They give an NP-hardness proof and develop an efficient algorithm assuming that the number of priceable edges is constant. Later their algorithm was improved by Cabello [11].

Cardinal et al. [12] proved several positive approximation results for the Stackelberg Minimum Spanning Tree Game. In the same paper, they proved that the revenue maximization for this game is APX-hard and strongly NP-hard. We make use of their reduction in the proof of Theorem 3. Furthermore, Cardinal et al. [13] prove that this game remains NP-hard if the instances are planar graphs. However, the problem becomes polynomial-time solvable on graphs of bounded treewidth. Bilo et al. [5] consider the Stackelberg Minimum Spanning Tree Game for complete graphs.

Briest et al. [9] consider Stackelberg Games where the follower's optimization problem cannot be solved to optimality. Instead the follower uses a known approximation algorithm. They show that the Stackelberg Knapsack Game is NP-hard if the follower uses a greedy 2 -approximate algorithm, and derive a $2+\epsilon$ approximation algorithm. Furthermore, the revenue maximization problem can be solved efficiently in the Stackelberg Vertex Cover Game if the follower implements a primal-dual approximation.

When there is more than one follower in the game, the so-called limited supply scenario naturally arises. In this scenario the additional assumption is made that the followers come in an order, and that an item can only be sold once and thus subsequent followers have a smaller supply of items to buy from. Balcan et al. [3] analyze the single-price strategy also in this setting and show that it yields a revenue of at least

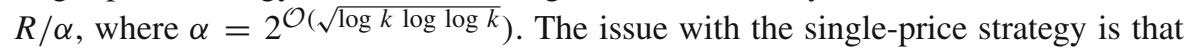
the same single-price is chosen for every follower, and this is difficult to control in the limited supply setting. Chakraborty et. al. [14] introduce a dynamic single-pricing strategy where a possibly different single-price is chosen for every follower. They are able to show that this modified strategy yields revenue of at least $R / \beta$, where $\beta=\mathcal{O}\left(\log ^{2} k\right)$.

Guruswami et al. [15] and Aggarwal et al. [1] consider envy-free pricings to maximize revenue. An envy-free pricing ensures a stable allocation of items to the followers. They show that computing an envy-free pricing to maximize revenue is APX-hard for (i) limited supply when there are unit demand followers and (ii) unlimited supply when there are single-minded followers. Unit demand followers buy no more than one item and single-minded followers are interested in only one subset of items. Guruswami et al. then give logarithmic approximation algorithms for both problems using a singleprice strategy in the unlimited supply case. 
A generalization of envy-pricing problems and Stackelberg matroid problems is studied in the context of assortment optimization by Berbeglia and Joret [4]. They use single price strategies to establish logarithmic approximation ratios.

\section{Approximability of Stackelberg Pricing Games}

In this section we first introduce the model in its full generality. Then we give a tight approximation result on the maximum revenue using a single-price strategy. We complement this with a hardness proof by showing that deciding whether one can achieve a revenue that is only slightly larger than the one guaranteed by the singleprice strategy is strongly NP-hard.

Our model Let $k$ and $n$ be some natural numbers with $k \leq n$. The optimization problem of the follower is the following: He minimizes a continuous function $f: \mathbb{R}^{n} \rightarrow \mathbb{R}$ over his set of feasible solutions $X \subseteq \mathbb{R}_{\geq 0}^{k} \times \mathbb{R}_{\geq 0}^{n-k}$. The only restriction we put on $X$ is that we require it to be a compact set, i.e., bounded and closed under limits.

The first move of the Stackelberg Pricing Game is made by the leader: She chooses a price vector $p \in \mathbb{R}^{k}$. Now the second move is made by the follower: He chooses an optimal solution $\left(x^{*}, y^{*}\right)$ of the program

$$
\begin{aligned}
& \min p^{T} x+f(x, y) \\
& \text { s.t. }(x, y) \in X .
\end{aligned}
$$

The revenue of the leader is then given by the value $p^{T} x^{*}$. This value is her objective function and it is to be maximized. We remark that this problem has a bilevel structure.

To avoid technicalities, we make the following optimistic assumption: If the follower has several optimal solutions in $X$, we assume that the solution which is most profitable for the leader is chosen. That is the solution, which maximizes the value $p^{T} x^{*}$. Moreover, we assume that there is a point $(x, y) \in X$ with $x$ being the $k$ dimensional all-zeroes vector. This simply means that the follower has a solution that does not give any revenue to the leader. Otherwise the revenue maximization problem would be unbounded which is obviously not an interesting case.

Before we can state our results for the new model, we need to introduce a number of technical notions. Given a feasible solution $(x, y) \in X$ of the follower, we call the value $\mathbf{1}^{T} x=\sum_{i=1}^{k} x_{i}$ the mass of $(x, y)$. A single-price is a price vector $p$ of the form $p=\lambda \mathbf{1}$ where $\lambda$ is some real number. Slightly abusing notation, we sometimes call $\lambda$ the single-price. Note that when the leader uses a single-price the revenue is simply the mass of the follower's solution times the single-price.

Let $M$ be the maximum mass the follower buys if the leader sets all her prices to 0. Formally,

$$
\begin{aligned}
M:=\max & \mathbf{1}^{T} x \\
\text { s.t. } & \exists y \in \mathbb{R}^{n-k}:(x, y)=\operatorname{argmin}\left\{f\left(x^{\prime}, y^{\prime}\right):\left(x^{\prime}, y^{\prime}\right) \in X\right\} .
\end{aligned}
$$

This value $M$ exists since $X$ is a compact set. 
Consider, for example, the case where the follower seeks to buy a shortest $s$ - $t$-path in a network. Then $M$ is the maximum number of priceable edges of a shortest $s$ - $t$-path in the network, when the priceable edges all have a price of 0 and thus can be bought for free by the follower.

Since $X$ is a compact set, there exists a largest single-price at which the follower buys a non-zero mass from the leader. Let $\mu$ be the maximum mass the follower buys at this price. Consider again the case where the follower searches for a shortest $s$ - $t$ path in a network. Then $\mu$ is the maximum number of priceable edges contained in a shortest $s$ - $t$-path, under the largest single-price for which a shortest path exists that contains a priceable edge.

For all $m \in[0, M]$, let $\Delta(m)$ be the minimum price the follower has to pay if he buys a mass of at most $m$ from the leader. More formally

$$
\begin{aligned}
\Delta(m):=\min & f(x, y) \\
\text { s.t. } & \mathbf{1}^{t} x \leq m \\
& (x, y) \in X,
\end{aligned}
$$

where $\mathbf{1}^{t} x$ is the mass bought by the follower. This minimum price $\Delta(m)$ exists, because $X$ with the additional constraint of $\mathbf{1}^{t} x \leq m$ is again a compact set.

As observed by several authors (cf. [3,8]), an upper bound on the optimum revenue is $R:=\Delta(0)-\Delta(M)$. To see this, let $r^{*}$ be the maximum revenue, and let $\left(x^{*}, y^{*}\right)$ be the corresponding follower's solution. We have

$$
r^{*}+\Delta(M) \leq r^{*}+\Delta\left(\mathbf{1}^{T} x^{*}\right) \leq r^{*}+f\left(x^{*}, y^{*}\right) \leq \Delta(0),
$$

because $\Delta(0)$ is an upper bound on the objective value of the follower and $\Delta$ is nonincreasing. We remark that $R$ is indeed a tight upper bound, in the sense that there are examples of games where the maximum revenue equals $R$, e.g., the minimum spanning tree pricing problem described in the introduction.

As our first result shows, the maximum revenue of the leader is always reasonably close to $R$, unless the ratio $M / \mu$ is large. This is true even if the leader uses a singleprice strategy.

Theorem 1 There is a single-price for the Stackelberg Pricing Game over X whose revenue is at least

$$
\frac{R}{1+\ln \left(\frac{M}{\mu}\right)}
$$

This result extends previous work of Briest et al. [10] and Balcan et al. [3], who proved the above theorem in the combinatorial setting, i.e., for $X \subseteq\{0,1\}^{n}$.

Proof We start with some mathematical background needed throughout the proof. Let $F:[a, b] \rightarrow \mathbb{R}$ be a continuous function. The lower left Dini derivative of $F$, denoted $D_{-} F$, is defined, for all $x \in(a, b]$, by 


$$
D_{-} F(x)=\liminf _{h \rightarrow 0^{-}} \frac{F(x)-F(x+h)}{h} .
$$

Later we need to recover $F$ from its lower left Dini derivative. For this, we need some more concepts.

The following discussion, including Theorem 2, follows the exposition of Hagood and Thomson [17]. A covering relation $\beta$ is a set of pairs $(x,[s, t])$ with $s<t$ and $x \in[s, t]$. Moreover, $\beta$ is called a left full cover of an interval $[a, b]$ if for each $x \in[a, b]$ there is a positive number $\rho(x)$ such that all of the following assertions hold.

(a) Every pair $(a,[a, t])$ for which $a<t<a+\rho(a)$ belongs to $\beta$.

(b) Every pair $(b,[s, b])$ for which $b-\rho(b)<s<b$ belongs to $\beta$.

(c) For each $x \in(a, b)$ and $t$ satisfying $x-\rho(x)<t<x$ there exists a positive number $\eta(x, t)$ such that $(x,[s, t])$ belongs to $\beta$ whenever $x \leq s \leq x+\eta(x, t)$.

Let $G:[a, b] \rightarrow \mathbb{R}$ be a function. We define the lower left Dini integral of $G$ over $[a, b]$ by

$$
\text { (LD) } \underline{\int_{a}^{b}} G(x)=\sup _{\beta} \inf _{\pi \subseteq \beta} S(G, \pi) \text {, }
$$

where $\beta$ runs through all left full covers $\beta$ of $[a, b], \pi$ runs through all partitions of $[a, b]$ contained in $\beta$, and $S(G, \pi)$ is a Riemann sum. Here, a partition of $[a, b]$ is a subset of $\beta$ of the form $\pi=\left\{\left(x_{i},\left[t_{i}, t_{i+1}\right]\right)\right\}$ for all $i=1, \ldots, k$, where $a=t_{0}<$ $t_{1}<\cdots<t_{k}=b$.

Theorem 2 (Cf. Hagood and Thomson [17]) If $F$ is a continuous function that has a finite lower left Dini derivative $D_{-} F(x)$ at every point $x \in[a, b]$, where $a, b \in \mathbb{R}$, then

$$
\text { (LD) } \int_{a}^{b} D_{-} F(x) d x=F(b)-F(a) \text {. }
$$

For each $m \in(0, M]$, let $P(m)$ be the supremum of all single-prices for which the follower has an optimal solution with a mass of at least $m$ from the leader. As the next claim shows, this supremum is indeed a maximum.

Claim 1 At the single-price of $P(m)$, the follower has an optimal solution of a mass of at least $m$.

Proof For each $n \in \mathbb{N}$, let $p_{n}=P(m) \cdot(1-1 / n)$ and let $z_{n} \in X$ be an optimum solution of the follower under the single-price $p_{n}$. By choice of $p_{n}$, we may choose $z_{n}$ such that the mass among the priceable variables is at least $m$.

Consider the sequence $\left(z_{n}\right)_{n \in \mathbb{N}}$. Since $X$ is compact, there is a convergent subsequence $\left(z_{n}\right)_{n \in S}$ where $S$ is some infinite subset of $\mathbb{N}$. Again by compactness, the limit point $z$ of the sequence $\left(z_{n}\right)_{n \in M}$ is in $X$. Obviously, $z$ contains a mass of at least $m$ 
among the priceable variables. Moreover, since the objective function of the follower is continuous, $z$ is an optimal solution under the single-price $P(m)$. This completes the proof.

Let $T \subseteq(0, M]$ be the set of values $t$ for which the follower does not have an optimal solution, at a single-price $P(t)$, with a mass more than $t$. The set $T$ plays a key role in the remainder of this proof.

Claim 2 It holds that $\mu \in T, M \in T$ and $\mu=\min T$. For all $m \in(0, M]$ it holds that $\max _{t \in T \cap[m, M]} P(t)$ exists and $P(m)$ equals this value.

Proof The first part of the claim follows directly from the definition of $\mu$ and $M$ respectively. Pick any $m \in(0, M]$. Let $m^{\prime}$ be the maximum mass which the follower will buy at single-price $P(m)$. This maximum exists since $X$ is closed. We have $P\left(m^{\prime}\right)=P(m)$, and $m^{\prime} \in T$.

Recall that $\Delta(\mathrm{m})$ is defined as the price the follower has to pay for the non-priceable variables if he buys a mass of at most $m$ from the leader. Similar to the proof of Briest et al. [10] for the combinatorial setting, we next show that the functions $P$ and $\Delta$ are closely related. In our case, however, we have to deal with several difficulties that arise because we allow for non-discrete optimization problems.

Consider the lower convex hull $H$ of the point set $\{(m, \Delta(m)): 0 \leq m \leq M\}$. Let $\partial H$ be the lower border of $H$, and let $\hat{\Delta}:[0, M] \rightarrow \mathbb{R}$ be the function for which $(m, \hat{\Delta}(m)) \in \partial H$ for all $m \in[0, M]$. We remark that, since $\hat{\Delta}$ is convex and decreasing,

$$
D_{-} \hat{\Delta}(m)=\sup _{\ell<m} \frac{\hat{\Delta}(m)-\hat{\Delta}(\ell)}{m-\ell} \text {, for each } m \in(0, M] \text {. }
$$

Claim 3 Let $t \in T$. Then $(t, \Delta(t)) \in \partial H$ and the point $(t, \Delta(t))$ is not the convex combination of any two other points in $\partial H$.

Proof If $t=M$, the statement is clear. So, we proceed to the case that $t \neq M$. Note that at a fixed single-price $p$ and fixed mass $m$ the follower buys a solution of costs $\Delta(m)+m p$. Furthermore, at a single-price of $P(t)$ the follower buys a mass of value exactly $t$. Hence, it must be the case that

$$
\Delta(t)+t P(t) \leq \Delta(\ell)+\ell P(t) \text { for all } 0<\ell<t
$$

and

$$
\Delta(t)+t P(t)<\Delta(r)+r P(t) \text { for all } t<r \leq M .
$$

Consequently,

$$
\frac{\Delta(r)-\Delta(t)}{r-t}<\frac{\Delta(t)-\Delta(\ell)}{t-\ell} \text { for all } 0<\ell<t<r \leq M
$$


Hence, $(t, \Delta(t)) \in \partial H$. In fact, the point $(t, \Delta(t))$ is not the convex combination of any two other points in $\partial H$.

Now we know that the elements of $T$ yield points of the convex hull $\partial H$. We proceed by showing that these points are exactly the points of the convex hull.

Claim 4 Fix $m \in(0, M)$. If for all $r \in(m, M]$ it holds that

$$
D_{-} \hat{\Delta}(m)<\frac{\hat{\Delta}(r)-\hat{\Delta}(m)}{r-m},
$$

then $m \in T$, and $D_{-} \hat{\Delta}(m)=-P(m)$.

Proof Since $D_{-} \hat{\Delta}(m)<(\hat{\Delta}(r)-\hat{\Delta}(m)) /(r-m)$ for all $r \in(m, M]$, the point $(m, \Delta(m))$ lies in $\partial H$. Hence, $\Delta(m)=\hat{\Delta}(m)$. For the ease of notation, let $p=$ $-D_{-} \hat{\Delta}(m)$. By $(1)$, for each $\ell \in(0, m)$ we have

$$
\frac{\Delta(m)-\Delta(\ell)}{m-\ell} \leq \frac{\hat{\Delta}(m)-\hat{\Delta}(\ell)}{m-\ell} \leq-p .
$$

It follows that $\Delta(m)+m p \leq \Delta(\ell)+\ell p$. Thus at the single-price $p$ a solution contains no less than a mass of $m$. Moreover, by assumption, for each $r \in(m, M]$ we have that

$$
-p<\frac{\hat{\Delta}(r)-\hat{\Delta}(m)}{r-m} \leq \frac{\Delta(r)-\Delta(m)}{r-m} .
$$

It follows that $\Delta(m)+m p<\Delta(r)+r p$, and thus the solution at the single-price $p$ has a mass of exactly $m$. In particular, $m \in T$ and $p=P(m)$.

Claim 5 It holds that $D_{-} \hat{\Delta}(M)=-P(M)$.

Proof Like in the proof of Claim 4, at a single-price of $-D_{-} \hat{\Delta}(M)$ a solution contains no less than a mass of $M$. Since $M$ is the maximum mass a follower will ever buy, $-D_{-} \hat{\Delta}(M)=P(M)$.

Claim 6 Except for a set of measure 0, it holds for all $m \in(0, M)$ that $D_{-} \hat{\Delta}(m)=$ $-P(m)$.

Proof Let $m \in(0, M)$. If $m$ satisfies the assumptions of Claim 4, we are done. Otherwise if for all $\ell \in(0, m)$ and for some $r \in(m, M]$

$$
\frac{\hat{\Delta}(m)-\hat{\Delta}(\ell)}{m-\ell}<D_{-} \hat{\Delta}(m)=\frac{\hat{\Delta}(r)-\hat{\Delta}(m)}{r-m},
$$

then we consider $m$ as an exception. Such an exception is illustrated in Fig. 2. Note that there is no other exception in the interval $[m, r)$ since the segment right of $m$ is a straight line: For any $m^{\prime} \in(m, r),(2)$ cannot hold for all $\ell \in\left(0, m^{\prime}\right)$. We can now 


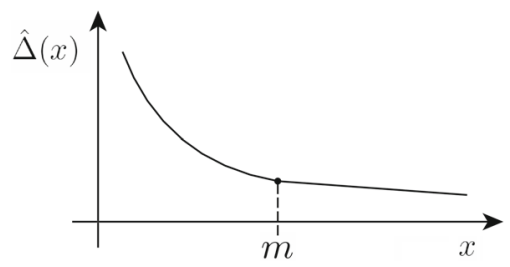

Fig. 2 The plot shows an exception as defined in (2) at the point $m$. The segment to the right of $m$ is a straight line and the Dini derivative is continuous at $m$

associate an exception with its interval. Since each such interval contains a rational number, the set of exceptions is countable and therefore has a measure of 0 .

Now we assume there are $\ell \in(0, m)$ and $r \in(m, M]$ for which

$$
\frac{\hat{\Delta}(m)-\hat{\Delta}(l)}{m-l}=D_{-} \hat{\Delta}(m)=\frac{\hat{\Delta}(r)-\hat{\Delta}(m)}{r-m} .
$$

Then for all $r^{\prime} \in(m, r]$ it holds that

$$
D_{-} \hat{\Delta}(m)=\frac{\hat{\Delta}\left(r^{\prime}\right)-\hat{\Delta}(m)}{r^{\prime}-m}
$$

Let $I \subseteq(m, M]$ be the set of values that satisfy (3). Then $I$ is a half-open interval of the form $I=\left(m, m^{\prime}\right]$. Note that $r \leq m^{\prime}$.

Observe that the segment $\left\{(x, \hat{\Delta}(x)): x \in\left[m, m^{\prime}\right]\right\}$ is a straight line. Hence,

$$
D_{-} \hat{\Delta}\left(m^{\prime}\right)=\liminf _{h \rightarrow 0^{-}} \frac{\hat{\Delta}\left(m^{\prime}\right)-\hat{\Delta}\left(m^{\prime}+h\right)}{h}=\frac{\hat{\Delta}\left(m^{\prime}\right)-\hat{\Delta}(m)}{m^{\prime}-m} \stackrel{(3)}{=} D_{-} \hat{\Delta}(m) .
$$

Assuming that $m^{\prime}<M$, we have

$$
D_{-} \hat{\Delta}\left(m^{\prime}\right)=D_{-} \hat{\Delta}(m)<\frac{\hat{\Delta}\left(r^{\prime}\right)-\hat{\Delta}(m)}{r^{\prime}-m} \leq \frac{\hat{\Delta}\left(r^{\prime}\right)-\hat{\Delta}\left(m^{\prime}\right)}{r^{\prime}-m^{\prime}} \text { for all } r^{\prime} \in\left(m^{\prime}, M\right] .
$$

The latter inequality holds because $\hat{\Delta}$ is a convex function. By Claim $4, m^{\prime} \in T$ and $D_{-} \hat{\Delta}\left(m^{\prime}\right)=-P\left(m^{\prime}\right)$. Since every point of the form $(x, \hat{\Delta}(x))$ is a convex combination of the two points $(\ell, \hat{\Delta}(\ell))$ and $\left(m^{\prime}, \hat{\Delta}\left(m^{\prime}\right)\right)$, for all $x \in\left[m, m^{\prime}\right)$, Claim 3 implies $T \cap\left[m, m^{\prime}\right)=\emptyset$. Hence,

$$
D_{-} \hat{\Delta}(m) \stackrel{(4)}{=} D_{-} \hat{\Delta}\left(m^{\prime}\right)=-P\left(m^{\prime}\right)=-P(m),
$$

by Claim 2 .

If $m^{\prime}=M$, then Claim 2 implies that $M \in T$, and Claim 5 implies $D_{-} \hat{\Delta}\left(m^{\prime}\right)=$ $-P\left(m^{\prime}\right)$. Like above, Claim 2, Claim 3 and (4) yield the desired statement. 
Now we are ready to give the calculation that finishes the proof. Using the fact that $\hat{\Delta}$ is continuous in combination with Theorem 2 , we see that

$$
\begin{aligned}
R & =\Delta(0)-\Delta(M)=\hat{\Delta}(0)-\hat{\Delta}(M)=\lim _{\epsilon \rightarrow 0^{+}} \hat{\Delta}(\epsilon)-\hat{\Delta}(M) \\
& =\lim _{\epsilon \rightarrow 0^{+}}(\mathrm{LD}) \int_{\epsilon}^{M}-D_{-} \hat{\Delta}(m) d m .
\end{aligned}
$$

Due to Claim 6, the integral

$$
\lim _{\epsilon \rightarrow 0^{+}}(\mathrm{LD}) \underline{\int_{\epsilon}^{M}} P(m) d m
$$

is well defined and equals

$$
\lim _{\epsilon \rightarrow 0^{+}}(\mathrm{LD}) \underline{\int_{\epsilon}^{M}}-D_{-} \hat{\Delta}(m) d m .
$$

Recall that, by Claim $2, \mu=\min T$ and so $P(m)=P(\mu)$ for all $m \in(0, \mu]$. Hence,

$$
\begin{aligned}
\lim _{\epsilon \rightarrow 0^{+}}(\mathrm{LD}) \underline{\int_{\epsilon}^{M}} P(m) d m & =\lim _{\epsilon \rightarrow 0^{+}}(\mathrm{LD}) \underline{\int_{\epsilon}} P(m) d m+(\mathrm{LD}) \int_{\mu}^{M} P(m) d m \\
& =\mu \cdot P(\mu)+(\mathrm{LD}) \underline{\int_{\mu}^{M}} P(m) d m .
\end{aligned}
$$

Let $r$ be the maximum revenue achieved by the single-price strategy. Note that $r$ is at least the revenue at the single-price $P(m)$, for each $m \in(0, M]$, which is in turn at least $m \cdot P(m)$. We thus have

$$
\begin{aligned}
\mu \cdot P(\mu)+(\mathrm{LD}) \int_{\mu}^{M} P(m) d m & =\mu \cdot P(\mu)+(\mathrm{LD}) \int_{\mu}^{M} \frac{m \cdot P(m)}{m} d m \\
& \leq r+(\mathrm{LD}) \int_{\mu}^{M} \frac{r}{m} d m \\
& =r+r \cdot(\ln (M)-\ln (\mu)) \\
& =r\left(1+\ln \left(\frac{M}{\mu}\right)\right) .
\end{aligned}
$$

This shows that $R \leq r(1+\ln (M / \mu))$, as desired.

Balcan et al. [3] and Briest et al. [10] also formulate an logarithmic approximation algorithm assuming that $R$ is known. With this assumption and the assumption that $M$ is known or computable, their approaches can be adapted to your setting. Define the single-prices $q_{l}=\frac{R}{2^{l-1}}$ for $l \in\{1, \ldots,\lceil\log (2 M)\rceil\}$. Balcan et al. show that picking one price $q_{l}$ uniformly at random yields an revenue with the logarithmic guarantee in 
expectation. Briest et al. formulate an algorithm that involves computing the revenue for all prices $q_{l}$ and selecting the single-price which yields the most revenue.

Moreover, Balcan et al. and Briest et al. extend their result to the situation when there are several followers. ${ }^{1}$ The same approach works in our generalized model.

Assume there are $\ell$ followers and each follower has its own optimization problem. Formally, the $i$-th follower minimizes his objective function $p^{T} x^{i}+f_{i}\left(x^{i}, y^{i}\right)$ where $\left(x^{i}, y^{i}\right)$ belongs to the set $X_{i} \subseteq \mathbb{R}_{\geq 0}^{k} \times \mathbb{R}_{\geq 0}^{n-k}$ of his feasible solutions, $i=1, \ldots, \ell$. The pricing vector $p$ appears in the objective function of every follower and is again set by the leader in order to maximize her revenue $\sum_{i=1}^{\ell} p^{T} x^{i}$. The difficulty here is that, while each follower has an individual optimization problem, the leader can set only one price vector for all followers at once. There is, however, a canonical way of reducing the pricing game to the case of a single follower. To this end, we consider the pricing game with respect to follower $i$ only, and let $\mu_{i}$ (resp. $M_{i}$ ) be the minimum non-zero mass (resp. the maximum mass) bought by follower $i$. Moreover, let $R_{i}$ be the upper bound on the revenue with respect to follower $i$.

Corollary 1 There is a single-price for the Stackelberg Pricing Game with $\ell$ followers whose revenue is at least

$$
\frac{\sum_{i=1}^{\ell} R_{i}}{1+\ln \left(\frac{\sum_{i=1}^{\ell} M_{i}}{\min _{i=1}^{\ell} \mu_{i}}\right)} .
$$

To see this, consider a single follower with the feasible subset $X=X_{1} \times X_{2} \times$ $\cdots \times X_{\ell}$. It is easy to see that we have $M=\sum_{i=1}^{\ell} M_{i}$ and $R=\sum_{i=1}^{\ell} R_{i}$ in this game. Moreover, the smallest non-zero mass $\mu$ bought by the newly defined single follower is at least the minimum smallest non-zero mass bought by one of the $\ell$ followers, that is $\min _{i=1}^{\ell} \mu_{i}$. Now applying Theorem 1 to the single follower yields Corollary 1 .

Theorem 1 is tight in the following sense.

Proposition 1 There are Stackelberg Pricing Games of arbitrarily large $R$ and $M$ in which the optimum revenue equals

$$
(1+o(1)) \cdot \frac{R}{1+\ln \left(\frac{M}{\mu}\right)} .
$$

This holds true even for games in which the follower minimizes a linear objective function of the form $p^{T} x+c^{T} y$ over a uniform matroid.

Proof Let $k \in \mathbb{N}$, let $X=\left\{x_{1}, \ldots, x_{k}\right\}$, and let $Y=\left\{y_{1}, \ldots, y_{k}\right\}$. Moreover, let $\mathcal{U}$ be the $k$-uniform matroid over the ground set $X \cup Y$. Finally, let $c_{i}=k / i$ be the cost of $y_{i}$ for each $i=1, \ldots, k$.

Consider the Stackelberg Pricing Game where the set $X$ is the set of priceable elements, and the follower seeks for a minimum cost basis of $\mathcal{U}$. Let us say $p_{i}$ is the price of the variable $x_{i}$ to be set by the leader.

\footnotetext{
1 In this paper we consider the case of unlimited supply, meaning that the followers buy their favorite solution independently of each other.
} 
Let $p_{1}^{*}, \ldots, p_{k}^{*}$ be optimal prices for the leader, where without loss of generality it holds that $p_{1}^{*} \leq \cdots \leq p_{k}^{*}$. Let $r$ be such that, under these prices, the follower buys exactly $r$ priceable elements. Of course, $r>0$.

Then it must hold that $p_{1}^{*} \leq \cdots \leq p_{r}^{*} \leq c_{r}=k / r$, and the revenue of the leader equals

$$
r^{*}=\sum_{i=1}^{r} p_{i}^{*} \leq k
$$

On the other hand, setting all prices to $k / r$ does also result in a solution where the follower buys $r$ priceable elements. Hence, we have $r^{*}=k$.

It is clear that $M=k$. Also, we have $\mu=1$, since a single-price of $k$ results in exactly one priceable element being bought by the follower. Moreover, we have

$$
R=\Delta(0)-\Delta(M)=\sum_{i=1}^{k} c_{i}-0=k \cdot H_{k},
$$

where $H_{k}$ is the $k$-th harmonic number. Hence,

$$
\lim _{k \rightarrow \infty} \frac{r^{*}}{\frac{R}{1+\ln \left(\frac{M}{\mu}\right)}}=\lim _{k \rightarrow \infty} \frac{1+\ln k}{H_{k}}=1,
$$

as desired.

Note that, in the above statement, every possible pricing is considered and not just single-price strategies. In other words, the lower bound in Theorem 1 is tight not only for single-price strategies, but for arbitrary pricings. So far, it was known that there are combinatorial pricing games where the optimum revenue is in $O(R / \log k)$, where $k$ is the number of priceable elements (cf. [8]). The merit of Proposition 1 is that it shows tightness of Theorem 1 up to a factor of $1+o(1)$, which is best possible. This fact, and the construction given in the proof of Proposition 1, enable us to prove the following hardness result.

Theorem 3 Fix a sufficiently small rational number $\epsilon>0$, and consider a Stackelberg Pricing Game where the follower minimizes an objective function of the form $p^{T} x+$ $c^{T} y$ over a matroid. It is strongly NP-hard to decide whether there is some pricing of revenue at least

$$
(1+\epsilon) \cdot \frac{R}{1+\ln \left(\frac{M}{\mu}\right)} .
$$

Proof Our reduction uses two ingredients: the hardness reduction of Cardinal et al. [12] for the Stackelberg Pricing Game on graphic matroids, and the uniform matroid discussed in Proposition 1. 

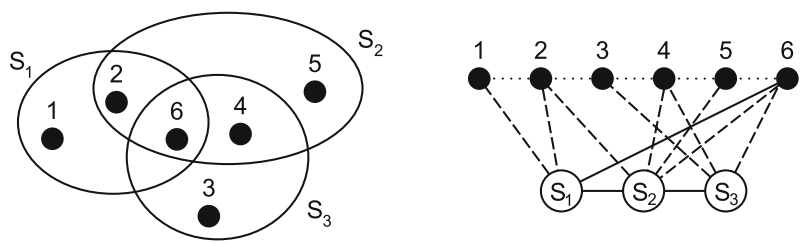

Fig. 3 A set cover instance is depicted on the left hand side with the corresponding Stackelberg Minimum Spanning Tree Game instance according to the reduction. The dashed edges are priceable. The dotted and straight edges have fixed cost of 1 and 2 respectively

Let us first recall the few details we need of the hardness reduction of Cardinal et al. [12]. For this, let $(\mathcal{S}, f)$ be an instance of the set cover problem, that is, $\mathcal{S}=\left\{S_{1}, \ldots, S_{m}\right\}$ is a finite family of finite sets and $f$ is a number. Let $\bigcup_{S \in \mathcal{S}} S=\left\{u_{1}, \ldots, u_{n}\right\}=: U$ and assume that $\mathcal{S}$ is disjoint from $U$. The well-known set cover problem is the problem of deciding whether there is a subfamily $\mathcal{S}^{\prime} \subseteq \mathcal{S}$ with both $\left|\mathcal{S}^{\prime}\right| \leq f$ and $\bigcup_{S \in \mathcal{S}^{\prime}} S=U$.

By introducing a dummy element, if necessary, we may assume that $u_{n}$ is contained in every set of $\mathcal{S}$. In the reduction, a graph $G$ on $m+n$ vertices is computed as follows. The vertex set of $G$ is $U \cup \mathcal{S}$, and every $u \in U$ is adjacent to some $S \in \mathcal{S}$ if and only if $u$ is contained in $S$. These edges are priceable, except for the edge $u_{n} S_{1}$ which has a fixed cost of 2. For each $i \in\{1, \ldots, m-1\}$, the edge $S_{i} S_{i+1}$ is contained in $G$, and all of these edges have a cost of 2 each. Moreover, for each $j \in\{1, \ldots, n-1\}$, the edge $u_{j} u_{j+1}$ is contained in $G$ and has a cost of 1 . The reduction is also illustrated in Fig. 3.

Consider now the problem of pricing the priceable edges of $G$ such that the revenue is maximized, where the follower computes a minimum spanning tree of $G$. As observed by Cardinal et al. [12], $(\mathcal{S}, f)$ is a yes-instance of the set cover problem if and only if there is a pricing achieving a revenue of at least $2 m+n-f-1$. In particular, the Stackelberg Minimum Spanning Tree Game is strongly NP-hard.

Our hardness reduction is again from the set cover problem, and the first step in our reduction is to perform exactly the same reduction as Cardinal et al. [12] described above. We may restrict our attention to the case of $m$ and $n$ being sufficiently large in a sense we define below, and we may also assume that $f \leq m$. Moreover, we may assume that the graph induced by the priceable edges is connected, which is equivalent to the fact that $S_{1}$ has a non-empty intersection with one of the sets $S_{2}, \ldots, S_{m}$. Let $\mathcal{G}$ be the graphical matroid ${ }^{2}$ of $G$, where the elements corresponding to fixed-cost edges of $G$ carry the same cost in $\mathcal{G}$.

Like in proof of Proposition 1, let $k \in \mathbb{N}$, let $X=\left\{x_{1}, \ldots, x_{k}\right\}$, and let $Y=$ $\left\{y_{1}, \ldots, y_{k}\right\}$. Moreover, let $\mathcal{U}$ be the $k$-uniform matroid over the ground set $E=X \cup Y$. Finally, let $c_{i}=k / i$ be the costs of $y_{i}$ for each $i=1, \ldots, k$.

Let $\mathcal{M}=\mathcal{U} \oplus \mathcal{G}$ be the direct sum of $\mathcal{U}$ and $\mathcal{G}$. That is, $\mathcal{M}$ is the unique matroid on the ground set that is the union of the ground sets of $\mathcal{U}$ and $\mathcal{G}$ and whose bases are exactly the Cartesian products of the bases of $\mathcal{U}$ and $\mathcal{G}$.

\footnotetext{
${ }^{2}$ Recall that the bases of a graphical matroid of a graph $H$ are the minimum spanning forests of $H$.
} 
Fix any $\epsilon$ with $0<\epsilon<1$. Consider the Stackelberg Pricing Game where the leader sets prices and the follower optimizes over $\mathcal{M}$. Denote the optimal revenue of the leader by $r_{\mathcal{M}}^{*}$. We will now show that if $k$ is chosen appropriately,

$$
r^{*} \geq(1+\epsilon) \cdot \frac{R_{\mathcal{M}}}{1+\ln \left(\frac{M_{\mathcal{M}}}{\mu_{\mathcal{M}}}\right)} \text { holds if and only if }(\mathcal{S}, f) \text { is a yes-instance }
$$

for the set cover problem. In (5), $R_{\mathcal{M}}, M_{\mathcal{M}}$ and $\mu_{\mathcal{M}}$ refer to the pricing problem over $\mathcal{M}$. In order to achieve a polynomial running time of the overall procedure, we need to choose $k$ such that it can be computed in polynomial time and its value is polynomially large in the value $n+m$.

In order to prove (5), let us first compute the actual value of $R_{\mathcal{M}} /\left(_{1}+\right.$ $\left.\ln \left(M_{\mathcal{M}} / \mu_{\mathcal{M}}\right)\right)$. It is clear that $R_{\mathcal{M}}$ is the sum of the respective upper bound $R_{\mathcal{G}}$ for the minimum spanning tree pricing problem and the respective upper bound $R_{\mathcal{U}}$ for the pricing problem over the uniform matroid. From the discussion in the last section we already know that $R_{\mathcal{U}}=k \cdot H_{k}$.

To compute $R_{\mathcal{G}}$, recall that the subgraph induced by the priceable edges in $G$ is connected, and this subgraph is spanning in $G$. Hence, there is a solution of the follower that only contains priceable edges, which in turn implies $\Delta_{\mathcal{G}}\left(M_{\mathcal{G}}\right)=0$. Furthermore, by construction of $G$ the solution of the follower avoiding all priceable edges uses exactly the non-priceable edges, which yields $\Delta_{\mathcal{G}}(0)=2 m+n-1$ in view of the fixed costs. Thus, $R_{\mathcal{G}}=2 m+n-1$ and so

$$
R_{\mathcal{M}}=R_{\mathcal{U}}+R_{\mathcal{G}}=k \cdot H_{k}+2 m+n-1 .
$$

To compute $M_{\mathcal{M}}$, we observe that at a single-price of 0 the follower buys $m+n-1$ many priceable edges in $\mathcal{G}$. Similarly, at a single-price of 0 the follower buys $k$ many priceable elements in $\mathcal{U}$. Together, we obtain

$$
M_{\mathcal{M}}=M_{\mathcal{U}}+M_{\mathcal{G}}=k+m+n-1 .
$$

Finally, to compute $\mu_{\mathcal{M}}$, note that, in $\mathcal{G}$, the follower does not buy any edge of the leader if the single-price is above 2 . Moreover, the follower buys exactly one edge at a single-price of $k$ in the pricing game over $\mathcal{U}$. As we will see below, $k>2$, and so we have

$$
\mu_{\mathcal{M}}=\mu_{\mathcal{U}}=1
$$

Summing up, we have

$$
\frac{R_{\mathcal{M}}}{1+\ln \left(\frac{M_{\mathcal{M}}}{\mu_{\mathcal{M}}}\right)}=\frac{k \cdot H_{k}+2 m+n-1}{1+\ln (k+m+n-1)} .
$$


We now want to choose $k$ such that

$$
k+2 m+n-f-1 \geq(1+\epsilon) \cdot \frac{R_{\mathcal{M}}}{1+\ln \left(\frac{M_{\mathcal{M}}}{\mu_{\mathcal{M}}}\right)}>k+2 m+n-f-2 .
$$

For a moment, let us assume that (7) holds for $k$. Consider the optimal revenue $r_{\mathcal{M}}^{*}$ of the pricing game over $\mathcal{M}$. Since $\mathcal{M}$ is the direct sum of $\mathcal{U}$ and $\mathcal{G}$, we have $r_{\mathcal{M}}^{*}=k+r_{\mathcal{G}}^{*}$, where $r_{\mathcal{G}}^{*}$ is the optimal revenue of the pricing game over $\mathcal{G}$. Recall that $(\mathcal{S}, f)$ is a yesinstance for the set cover problem if and only if $r_{\mathcal{G}}^{*} \geq 2 m+n-f-1$. Consequently, $(\mathcal{S}, f)$ is a yes-instance if and only if $r_{\mathcal{M}}^{*} \geq k+2 m+n-f-1$. Since $r_{\mathcal{G}}^{*}$ and thus also $r_{\mathcal{M}}^{*}$ are both integers, (7) implies that

$$
r_{\mathcal{M}}^{*} \geq k+2 m+n-f-1 \text { if and only if } r_{\mathcal{M}}^{*} \geq(1+\epsilon) \cdot \frac{R_{\mathcal{M}}}{1+\ln \left(\frac{M_{\mathcal{M}}}{\mu_{\mathcal{M}}}\right)}
$$

In turn, (5) holds, and gives the desired hardness result.

We now show that $k$ can be chosen, and efficiently computed, such that (7) holds. Note that, assuming $k=\Omega(m+n)$,

$$
\frac{1}{k} \cdot \frac{k \cdot H_{k}+2 m+n-1}{1+\ln (k+m+n-1)}=\frac{H_{k}+O(1)}{\ln (k)+O(1)} \stackrel{m+n \rightarrow \infty}{\longrightarrow} 1 .
$$

In other words, assuming $k=\Omega(m+n)$,

$$
\frac{k \cdot H_{k}+2 m+n-1}{1+\ln (k+m+n-1)}=(1+o(1)) \cdot k \quad \text { when } m+n \text { grows. }
$$

Now let us put $k^{\prime}=\lceil 4(2 m+n-f-1) / \epsilon\rceil$ and $k^{\prime \prime}=\lfloor(2 m+n-f-2) /(4 \epsilon)\rfloor$. Then $k^{\prime}+2 m+n-f-1 \leq(1+\epsilon / 2) k^{\prime}$ and $k^{\prime \prime}+2 m+n-f-2 \geq(1+2 \epsilon) k^{\prime \prime}$, assuming $m+n$ is large enough. Hence, by applying (8) with respect to $k^{\prime}$,

$$
k^{\prime}+2 m+n-f-1 \leq(1+\epsilon / 2) k^{\prime} \stackrel{(8)}{<}(1+\epsilon) \cdot \frac{k^{\prime} \cdot H_{k^{\prime}}+2 m+n-1}{1+\ln \left(k^{\prime}+m+n-1\right)}
$$

if $m+n$ is large enough. Similarly,

$$
k^{\prime \prime}+2 m+n-f-2 \geq(1+2 \epsilon) k^{\prime \prime} \stackrel{(8)}{>}(1+\epsilon) \cdot \frac{k^{\prime \prime} \cdot H_{k^{\prime \prime}}+2 m+n-1}{1+\ln \left(k^{\prime \prime}+m+n-1\right)}
$$

if $m+n$ is large enough. From now on we assume $m+n$ to be large enough for all above inequalities to hold.

We now show that we can choose $k \in\left[k^{\prime \prime}, k^{\prime}\right]$ such that (7) holds. To see this, fix $\delta>0$ such that $\delta+\epsilon<1$. Note that, since $k \in\left[k^{\prime \prime}, k^{\prime}\right]$, we have $k=\Omega(m+n)$. By (6) and (8), an increase of $k$ by 1 leads to an increase of $(1+\epsilon) \cdot R_{\mathcal{M}} /\left(1+\ln \left(M_{\mathcal{M}} / \mu_{\mathcal{M}}\right)\right)$ by at most $1+\epsilon+\delta$, provided $m+n$ is large enough. Consequently, an increase of $k$ by 
1 leads to an increase of $(1+\epsilon) \cdot R_{\mathcal{M}} /\left(1+\ln \left(M_{\mathcal{M}} / \mu_{\mathcal{M}}\right)\right)-k$ by at most $\epsilon+\delta<1$, where we assume that $m+n$ is large enough. This, together with (9) and (10), shows that there is some $k \in\left[k^{\prime \prime}, k^{\prime}\right]$ as desired.

Moreover, it is possible to find such a $k$ in polynomial time. This is due to the fact that $\epsilon$ is a rational constant, $f \leq m$ by assumption, $k^{\prime}, k^{\prime \prime}=O(m+n)$, and that a polynomial number of first digits of the natural logarithm can be computed in polynomial time (cf. [7]). This completes the proof.

In the statement of the above theorem, we assume that the matroid is given by its ground set and a membership oracle. Thus, $\mu, M$, and $R$ can be computed in polynomial time. Moreover, it will be clear from the proof that the natural logarithm can be computed in polynomial time with the precision needed to decide the problem.

Let us remark that the assumption of $\epsilon$ being sufficiently small is merely technical, and can be dropped by giving a more careful hardness reduction. The message of the above theorem is, however, that there is a sharp contrast between the revenue guaranteed by the simple single-price strategy given in Theorem 1 and anything more than that.

\section{Parameterized complexity of Stackelberg Pricing Games with few priceable objects}

In this section we study the parameterized complexity of Stackelberg Pricing Games when parameterized by the number of priceable objects. Intuitively, this addresses the question of whether there are improved algorithms for the case that only few objects are priceable. We give a general positive result for the combinatorial model. Our main result in this part, however, is a hardness proof for the linear programming case; we begin with the latter.

In the LP- PRICING problem there is a linear program over which the follower minimizes. The leader may choose the price, i.e. target function coefficient, of $k$ specified variables. Her revenue is determined by the corresponding (weighted) sum over these variables.

LP- PRICING

Input: A linear program with $k$ priceable variables and $\lambda \in \mathbb{Q}$.

Question: Is there a price vector whose revenue is at least $\lambda$ ?

We prove that this problem is at least as hard to solve as the parameterized $k$-clique problem. The hardness proof creates linear programs with only non-negative variables and non-negative target function over which the follower seeks to minimize. As such it proves hardness also for our more general model parameterized by number of priceable variables.

Theorem 4 LP- PRICING is W[1]-hard when parameterized by the number $k$ of priceable variables.

The theorem is proven by a reduction from the well-known (parameterized) MULTICOLORED CLIQUE problem. Therein, we are given a $k$-partite graph $G$ (or, equivalently, 
a properly $k$-colored graph) and have to determine whether $G$ contains a clique on $k$ vertices; the problem is W[1]-hard with respect to parameter $k$. Thus, unless FPT $=\mathrm{W}[1]$, there is no algorithm running in time $f(k) n^{c}$ for instances of size $n$. Moreover, under the Exponential-Time Hypothesis [18] the reduction implies that there is no $O\left(n^{o(k)}\right)$ time algorithm for LP- PRICING. In instances created by the reduction the leader can effectively enforce the choice of the $k$ clique vertices by setting appropriate prices for $k$ variables; the remaining variables are used to verify the choice and a certain revenue threshold can only be attained if there is indeed a $k$-clique. The behavior of these $k$ priceable variables is quite similar to integer variables, as they can be shown to only take specific values from a finite set in solutions meeting the threshold (one value corresponding to each vertex of $G$ ). This arguably gives our parameterized LP PRICING problem some similarity to the MIXED ILP FEASIBILITY problem parameterized by the number of integer variables. Interestingly, the latter problem is FPT due to a classic result of Lenstra [16].

Proof We give a parameterized reduction from the W[1]-hard MULTICOLORED CLIQUE $(k)$ problem. Therein, we are given a $k$-partite graph and have to determine whether it contains a clique of size $k$, i.e., a clique containing exactly one vertex from each partite set. The reduction will be polynomial-time computable and increases the parameter value by exactly one, proving W[1]-hardness of LP- PRICING.

Construction. Let $(G, k)$ an instance of MULTICOLORED CLIQUE $(k)$ where $G=$ $\left(V_{1}, \ldots, V_{k}, E\right)$. Without loss of generality assume that each set $V_{i}$ contains the same number $n$ of vertices, and that $V_{i}=\{(i, 1), \ldots,(i, n)\}$. Similarly, we may assume that $n \geq 12$. We now describe the construction of a linear program, beginning with the used variables:

- Variables $x_{0}, x_{1}, \ldots, x_{k}$ which are priceable. The corresponding base costs in the LP are zero, but the leader may specify additional prices $d_{0}, d_{1}, \ldots, d_{k}$. All further variables are not priceable and only have a base cost.

- Variables $y_{0}, y_{1}, \ldots, y_{k}$. The base cost for $y_{0}$ is $c_{0}$; for $y_{1}, \ldots, y_{k}$ it is $c_{y}=2$. Together with $x_{1}, \ldots, x_{k}$ the latter $k$ variables correspond to choosing the vertices of a (possible) $k$-clique. Variables $x_{0}$ and $y_{0}$ provide slack for the clique constraints that we give later, and full leader payoff can only be attained when no slack is needed.

- Variables $w_{i, 1}, \ldots, w_{i, n}$ for each $i \in[k]$. These are used to ensure that the leader cannot make profit more than 1 with any variable $x_{i}$, i.e., that $d_{i} x_{i} \leq 1$. This is nontrivial to achieve since the profit with $x_{i}$ depends on the (variable) price $d_{i}$ whereas we need to have fixed prices for the $w_{i, \ell}$ (to be detailed later).

- Variables $z_{i, 1}, \ldots, z_{i, n}$ for each $i \in[k]$. These play the role of indicator variables, with the caveat of being fractional. They all have the same $\operatorname{cost} c_{z}=\frac{1}{n}$.

A central part of the construction is to effectively allow the leader to choose the value of $x_{i}$ and $y_{i}$ in optimal solutions for the follower by specifying an appropriate price $d_{i}$ for $x_{i}$. At high level, there will be a set of linear constraints on $x_{i}$ and $y_{i}$ that are defined by convex set of points (so the feasible points for $x_{i}$ and $y_{i}$ are above a piecewise linear convex function spanned by the points). The points (in two dimensions) are $\left(p_{1}, q_{1}\right), \ldots,\left(p_{n+1}, q_{n+1}\right)$ where 


$$
p_{j}=2^{-j} \quad q_{j}=\sum_{\ell=0}^{j-1} \delta_{\ell} \quad \delta_{j}=\frac{n}{2 n+4 j} .
$$

Note that $q_{0}=0$ and $q_{j+1}=q_{j}+\delta_{j}$. Similarly, we have $p_{1}=\frac{1}{2}$ and $p_{j+1}=$ $p_{j}-2^{-j-1}=2^{-j-1}$. Note that point $\left(p_{n+1}, q_{n+1}\right)$ is only needed for technical reasons (e.g., defining a constraint) and is not intended as a choice for $\left(x_{i}, y_{i}\right)$. The intended price for $\left(x_{i}, y_{i}\right)=\left(p_{j}, q_{j}\right)$ for $j \in\{1, \ldots, n\}$ will be

$$
r_{j}=2^{j}
$$

Quite a bit of this proof will go towards showing that using $d_{i} \in\left\{r_{1}, \ldots, r_{n}\right\}$ is the only way for the leader to achieve the target payoff of at least $k+c_{0}$.

We will continue with establishing (strict) convexity of the point sequence $\left(p_{1}, q_{1}\right), \ldots$, $\left(p_{n+1}, q_{n+1}\right)$.

Claim 7 The point sequence $\left(p_{1}, q_{1}\right), \ldots,\left(p_{n+1}, q_{n+1}\right)$ is strictly convex.

Proof Consider the sequence of slopes

$$
\frac{p_{2}-p_{1}}{q_{2}-q_{1}}, \frac{p_{3}-p_{2}}{q_{3}-q_{2}}, \ldots, \frac{p_{n}-p_{n-1}}{q_{n}-q_{n-1}}, \frac{p_{n+1}-p_{n}}{q_{n+1}-q_{n}} .
$$

It suffices to show that $\frac{p_{j+1}-p_{j}}{q_{j+1}-q_{j}}<\frac{p_{j+2}-p_{j+1}}{q_{j+2}-q_{j+1}}$ for all $j \in\{1, \ldots, n-1\}$, i.e., that the slopes between consecutive points are strictly increasing. We have

$$
\begin{aligned}
& \frac{p_{j+1}-p_{j}}{q_{j+1}-q_{j}}<\frac{p_{j+2}-p_{j+1}}{q_{j+2}-q_{j+1}} \\
& \Leftrightarrow \quad \frac{-2^{-j-1}}{\delta_{j}}<\frac{-2^{-j-2}}{\delta_{j+1}} \\
& \Leftrightarrow \quad \frac{2^{-j-1}}{\delta_{j}}>\frac{2^{-j-2}}{\delta_{j+1}} \\
& \Leftrightarrow \quad 2 \delta_{j+1}>\delta_{j} \\
& \Leftrightarrow \quad \frac{2 n}{2 n+4(j+1)}>\frac{n}{2 n+4 j} \\
& \Leftrightarrow \quad 2 n \cdot(2 n+4 j)>n \cdot(2 n+4 j+4) \\
& \Leftrightarrow \quad 4 n^{2}+8 n j>2 n^{2}+4 n j+4 n \\
& \Leftrightarrow \quad 2 n^{2}+4 n j-4 n>0 \\
& \Leftrightarrow \quad 2 n^{2}+4 n(j-1)>0 \text {. }
\end{aligned}
$$

We use that $\delta_{j}, \delta_{j+1}>0$, that $n \geq 1$, and that $j \geq 1$, where the latter two imply that the final inequality holds, proving (by equivalence) the claimed strict increase in slopes. This completes the proof of the claim.

We will now give a set of line equations on variables $x$ and $y$, indexed by $j \in\{1, \ldots, n\}$, such that equation $j$ captures exactly the line through points 
$(x, y)=\left(p_{j}, q_{j}\right)$ and $(x, y)=\left(p_{j+1}, q_{j+1}\right)$. These will later be the basis for the core constraints on pairs $x_{i}$ and $y_{i}$ of variables (for $i \in[k]$ ). Consider the following line equation.

$$
x+p_{j+1} \frac{1}{\delta_{j}} y=p_{j}+p_{j+1} \frac{1}{\delta_{j}} q_{j}
$$

Clearly, $(x, y)=\left(p_{j}, q_{j}\right)$ fulfills the equation. For $(x, y)=\left(p_{j+1}, q_{j+1}\right)$ consider that

$$
\begin{aligned}
x+p_{j+1} \frac{1}{\delta_{j}} y & =p_{j+1}+p_{j+1} \frac{1}{\delta_{j}} q_{j+1} \\
& =p_{j+1}+p_{j+1} \frac{1}{\delta_{j}}\left(\delta_{j}+q_{j}\right) \\
& =p_{j+1}+p_{j+1}+p_{j+1} \frac{1}{\delta_{j}} q_{j} \\
& =p_{j}+p_{j+1} \frac{1}{\delta_{j}} q_{j},
\end{aligned}
$$

using $p_{j+1}+p_{j+1}=2 \cdot 2^{-j-1}=2^{-j}=p_{j}$. Thus, the two claimed points fulfill (13).

Consider now the constraint obtained from (13):

$$
x+p_{j+1} \frac{1}{\delta_{j}} y \geq p_{j}+p_{j+1} \frac{1}{\delta_{j}} q_{j}
$$

It is fulfilled to equality for $(x, y) \in\left\{\left(p_{j}, q_{j}\right),\left(p_{j+1}, q_{j+1}\right)\right\}$. Since the points $\left(p_{1}, q_{1}\right), \ldots,\left(p_{n+1}, q_{n+1}\right)$ are in strictly convex position (as a function $x=x(y)$ with $\left.(x, y) \in\left\{\left(p_{1}, q_{1}\right), \ldots,\left(p_{n+1}, q_{n+1}\right)\right\}\right)$ the constraint is fulfilled to $>$ for any $(x, y) \in\left\{\left(p_{1}, q_{1}\right), \ldots,\left(p_{j-1}, q_{j-1}\right),\left(p_{j+2}, q_{j+2}\right), \ldots,\left(p_{n+1}, q_{n+1}\right)\right\}$. For ease of reference we will make this an explicit (and just established) claim.

Claim 8 Let $(x, y) \in\left\{\left(p_{1}, q_{1}\right), \ldots,\left(p_{n}, q_{n}\right)\right\}$. Then $(x, y)$ fulfills (14) for every $j \in\{1, \ldots, n-1\}$ and it is fulfilled to equality if and only if $(x, y) \in$ $\left\{\left(p_{j}, q_{j}\right),\left(p_{j+1}, q_{j+1}\right)\right\}$.

In addition to adding constraints (14) for all pairs $\left(x_{i}, y_{i}\right)$, with some small modifications, we will also require $y_{i} \geq 0$ and $x_{i} \geq 0$. Together with constraints of type (14) this gives the following extremal points in two dimensions (considering only $x_{i}$ and $\left.y_{i}\right)$, each defined by two tight constraints: $\left(p_{1}, q_{1}\right)=\left(p_{1}, 0\right),\left(p_{2}, q_{2}\right), \ldots$, $\left(p_{n}, q_{n}\right)$, and $\left(0, q_{n}+2 \delta_{n}\right)=\left(0, q_{n+1}+\delta_{n}\right)$. In other words, we could also have used $\left(0, q_{n}+2 \delta_{n}\right)$ to define (14) for $j=n$ as this is the intersection point of the supporting line with the line $x=0$. It is somewhat more intuitive to use $\left(0, q_{n}+2 \delta_{n}\right)$ rather than $\left(p_{n+1}, q_{n+1}\right)$, which is not defined by two tight constraints, but that would always warrant a special treatment. 
Let us recall the high level idea of forcing the follower to use $\left(x_{i}, y_{i}\right)=\left(p_{j}, q_{j}\right)$ when $d_{i}=r_{j}$; this will create a leader payoff of exactly 1 for $x_{i}$, since $d_{i} x_{i}=r_{j} p_{j}=1$. Unfortunately, the problem setting does not allow for us to specify a set of permissible leader prices and, modulo further constraints that we did not specify yet, we do not know yet that the follower will react in the desired way. It turns out that part of the issues can be resolved if we can ensure that the leader can have profit at most 1 per variable $x_{i}$. (E.g. this will ensure that a valid solution to the pricing problem will have to make another minuscule amount of profit on $x_{0}$, rather than making this amount on some $x_{i}$ 's.)

The basic idea for limiting profit at $x_{i}$ is to create an additional variable, say $w_{i}$, (not priceable) with fixed price and putting in each constraint $x_{i}+w_{i}$ instead of $x_{i}{ }^{3}$ Accordingly, if the leader price exceeds the price of $w_{i}$ then the follower would set $x_{i}=0$ and instead increase $w_{i}$. Note, however, that we do not have a single price level in mind for $x_{i}$, so this simple idea will not work.

Instead, we use $n$ different variables $w_{i, 1}, \ldots, w_{i, n}$ and let the price of $w_{i, \ell}$ be $r_{\ell}$, i.e., the intended price for $x_{i}=p_{\ell}$. Now of course we still need to be careful since e.g. $w_{i, 1}$ costs only $r_{1}=2$ whereas $x_{i}$ will typically be more expensive. Intuitively, since the constraints (14) for different values of $j$ effectively correspond to particular point $\left(p_{j}, q_{j}\right)$, which have intended prices $r_{j}$, we can introduce those variables $w_{i, \ell}$ whose intended prices $r_{\ell}$ exceed $r_{j}$, i.e., those with $\ell \geq j$. Without further ado, we get the following core constraints for $j \in[n]$ :

$$
x_{i}+\sum_{\ell=j}^{n} w_{i, \ell}+p_{j+1} \frac{1}{\delta_{j}} y_{i} \geq p_{j}+p_{j+1} \frac{1}{\delta_{j}} q_{j}
$$

The remaining constraints are comparatively simple. (We remark, nevertheless, that the choice of $\left(p_{1}, q_{1}\right), \ldots,\left(p_{n}, q_{n}\right)$ depends in part on these constraints since they incur further costs, which the follower will balance against the amount paid to the leader.) For the (effective) indicator variables $z_{i, j}$ we ensure that

$$
z_{i, j} \geq\left|y_{i}-q_{j}\right|
$$

i.e., that they are at least equal to the absolute value of the difference between $y_{i}$ and $q_{j}$, by adding constraints

$$
z_{i, j} \geq y_{i}-q_{j}, \quad z_{i, j} \geq q_{j}-y_{i} .
$$

Note that a variable $z_{i, j}$ can only take value 0 if $y_{i}=q_{j}$. In that (limited) sense they are indicator variables for whether $y_{i}=q_{j}$. Using these variables we set up clique-testing constraints

$$
z_{i, u}+z_{j, v}+\varepsilon y_{0} \geq \varepsilon
$$

\footnotetext{
3 Variable $x_{i}$ will occur only positively and on the left hand side of $\geq$ constraints.
} 
for all $(i, u) \in V_{i}$ and $(j, v) \in V_{j}$ with $\{(i, u),(j, v) \notin E\}$, i.e., to prevent that both $(i, u)$ and $(j, v)$ are chosen for the clique. We let $\varepsilon=\delta_{n}=\frac{1}{6}$, but any sufficiently small value will do. (We do have to ensure that the constraint holds if $y_{i} \neq q_{u}$ or $y_{j} \neq q_{v}$.) In intended solutions variable $y_{0}$ will take value 0 , but the proof will make several replacement arguments where increasing $y_{0}$ to 1 ensures that we do not violate these constraints.

The final constraint is

$$
x_{0}+y_{0}=1
$$

which may seem a bit useless at first glance but it allows some important arguments about the leader profit. Recall that $x_{0}$ has base cost 0 and is priceable with price $d_{0}$, whereas $y_{0}$ has only a base cost of $c_{0}$, which is a small constant to be chosen later. Since $x_{0}$ appears nowhere else, the follower will set it to 0 if $d_{0}>c_{0}$ as increasing $y_{0}$ to 1 will not violate any constraint. If there is a solution that fulfills the clique constraints with $y_{0}=0$ then the leader can gain profit of up to $c_{0}$ by setting $d_{0}=c_{0}$.

This completes the LP. We have constraints of types (15), (17), (18), and (19). All variables are constrained to non-negative values. The target leader payoff is set to

$$
k+c_{0},
$$

with the intention of gaining exactly 1 per $x_{i}$ with $i \in[k]$ and $c_{0}$ for $x_{0}$.

Clearly, the LP can be generated in polynomial time. We have $k+1$ leader variables, so the new parameter value is indeed bounded by a function of the old one. (In fact this is tight enough to also inherit running time lower bounds from MULTICOLORED CLIQUE ( $k$ ) under ETH.) It remains to prove correctness.

(i) Existence of a $k$-clique implies solution with high leader payoff. To prove correctness, let us first assume that the input instance of MULTICOLORED CLIQUE $(k)$ is yes, and let $\left\{\left(1, v_{1}\right), \ldots,\left(k, v_{k}\right)\right\}$ be the vertices of a $k$-clique in $G$ (with exactly one vertex $v_{i}$ per partite set $V_{i}$ ). Define prices and a proposed solution for the linear program as follows:

- Let $d_{0}=c_{0}$ and let $d_{i}=r_{v_{i}}$ for all $i \in[k]$.

- Let $x_{0}=1$ and $y_{0}=0$.

- Let $\left(x_{i}, y_{i}\right)=\left(p_{v_{i}}, q_{v_{i}}\right)$ for all $i \in[k]$.

- Let $z_{i, j}=\left|y_{i}-q_{j}\right|$ for all $i \in[k]$ and $j \in[n]$.

- Let $w_{i, j}=0$ for all $i \in[k]$ and $j \in[n]$.

Clearly, for the given leader prices, this solution attains a leader payoff of exactly $d^{T} x=k+c_{0}$. It remains to verify feasibility and optimality of this solution for the follower.

(i.1) Feasibility. By construction of the core constraints (15) all of them are fulfilled by letting $\left(x_{i}, y_{i}\right)=\left(p_{v_{i}}, q_{v_{i}}\right)$. Clearly, the indicator constraints (17) are fulfilled by $z_{i, j}=\left|y_{i}-q_{j}\right|$. Obviously, $x_{0}=1$ and $y_{0}=0$ fulfills $x_{0}+y_{0}=1$, and all variables are non-negative. Let us consider the clique-testing constraints (18):

$$
z_{i, u}+z_{j, v}+\varepsilon y_{0} \geq \varepsilon
$$


If $v_{i} \neq u$ then $y_{i}=q_{v_{i}} \neq q_{u}$. This implies that $z_{i, u}=\left|q_{v_{i}}-q_{u}\right| \geq \delta_{n} \geq \varepsilon$ since any two $q_{j}$ values are at least $\delta_{n}$ apart. This already fulfills the constraint, and the analogue works if $v_{j} \neq v$. If $v_{i}=u$ and $v_{j}=v$ then, as $\left\{\left(1, v_{1}\right), \ldots,\left(k, v_{k}\right)\right\}$ is a clique in $G$, there must be an edge $\left\{\left(i, v_{i}\right),\left(j, v_{j}\right)\right\}$. Hence, there is no such constraint with $v_{i}=u$ and $v_{j}=v$. This completes feasibility of our proposed solution.

(i.2) Optimality. It remains to prove that the proposed solution attains the minimum possible follower cost, given the chosen prices $d_{0}, d_{1}, \ldots, d_{k}$. Let us first spell out the target function including the leader prices, skipping terms corresponding to variables with base cost 0 :

$$
T=d_{0} x_{0}+c_{0} y_{0}+\sum_{i=1}^{k} d_{i} x_{i}+\sum_{i=1}^{k} c_{y} y_{i}+\sum_{i=1}^{k} \sum_{j=1}^{n} c_{z} z_{i, j}+\sum_{i=1}^{k} \sum_{j=1}^{n} r_{j} w_{i, j}
$$

Plugging in the chosen prices and values for our proposed solution we directly obtain its cost for the follower:

$$
C=c_{0}+k+\sum_{i=1}^{k} 2 q_{v_{i}}+\sum_{i=1}^{k} \sum_{j=1}^{n} \frac{1}{n}\left|q_{v_{i}}-q_{j}\right|
$$

Now, let us return to the target function (20) and start deriving the lower bound. We will make a few simplifications and then, for readability, focus on the contribution for fixed $i \in[k]$. We begin with plugging in the known prices and using $x_{0}+y_{0}=1$.

$$
\begin{gathered}
T=d_{0} x_{0}+c_{0} y_{0}+\sum_{i=1}^{k} d_{i} x_{i}+\sum_{i=1}^{k} c_{y} y_{i}+\sum_{i=1}^{k} \sum_{j=1}^{n} c_{z} z_{i, j}+\sum_{i=1}^{k} \sum_{j=1}^{n} r_{j} w_{i, j} \\
=c_{0}+\sum_{i=1}^{k} r_{v_{i}} x_{i}+\sum_{i=1}^{k} 2 y_{i}+\frac{1}{n} \sum_{i=1}^{k} \sum_{j=1}^{n} z_{i, j}+\sum_{i=1}^{k} \sum_{j=1}^{n} r_{j} w_{i, j}
\end{gathered}
$$

Let us now focus on the contribution of variables $x_{i}, y_{i}, z_{i}$, , and $w_{i}$, to the target function, denoting this with $T(i)$ such that $T=c_{0}+\sum_{i=1}^{k} T(i)$, i.e.,

$$
T(i)=r_{v_{i}} x_{i}+2 y_{i}+\frac{1}{n} \sum_{j=1}^{n} z_{i, j}+\sum_{j=1}^{n} r_{j} w_{i, j}
$$

As a first step, let us derive a lower bound for $\sum_{j=1}^{n} r_{j} w_{i, j}$. With the goal of later applying constraint (15) for $j=v_{i}$ we seek to remove $w_{i, j}$ with $j<v_{i}$ and to get a uniform coefficient: 


$$
\begin{aligned}
\sum_{j=1}^{n} r_{j} w_{i, j} & =\sum_{j=1}^{v_{i}-1} r_{j} w_{i, j}+\sum_{j=v_{i}}^{n} r_{j} w_{i, j} \\
& \geq 0+\sum_{j=v_{i}}^{n} r_{v_{i}} w_{i, j} \\
& =r_{v_{i}} \sum_{j=v_{i}}^{n} w_{i, j}
\end{aligned}
$$

We use that $r_{j} \geq r_{v_{i}}$ for $j \geq v_{i}$ and that $r_{j} \geq 0$ and $w_{i, j} \geq 0$. We now insert this into (23) and get

$$
\begin{aligned}
T(i) & \geq r_{v_{i}} x_{i}+2 y_{i}+\frac{1}{n} \sum_{j=1}^{n} z_{i, j}+r_{v_{i}} \sum_{j=v_{i}} w_{i, j} \\
& =r_{v_{i}}\left(x_{i}+\sum_{j=v_{i}}^{n} w_{i, j}\right)+2 y_{i}+\frac{1}{n} \sum_{j=1}^{n} z_{i, j}
\end{aligned}
$$

We will now use constraint (15) for $j=v_{i}$ and first slightly transform it into a lower bound for $x_{i}+\sum_{j=v_{i}}^{n} w_{i, j}$ :

$$
\begin{gathered}
x_{i}+\sum_{\ell=v_{i}}^{n} w_{i, \ell}+p_{v_{i}+1} \frac{1}{\delta_{v_{i}}} y_{i} \geq p_{v_{i}}+p_{v_{i}+1} \frac{1}{\delta_{v_{i}}} q_{v_{i}} \\
\Leftrightarrow x_{i}+\sum_{\ell=v_{i}}^{n} w_{i, \ell} \geq p_{v_{i}}+p_{v_{i}+1} \frac{1}{\delta_{v_{i}}}\left(q_{v_{i}}-y_{i}\right)
\end{gathered}
$$

Inserting (26) into (25) yields

$$
\begin{aligned}
T(i) & \geq r_{v_{i}}\left(p_{v_{i}}+p_{v_{i}+1} \frac{1}{\delta_{v_{i}}}\left(q_{v_{i}}-y_{i}\right)\right)+2 y_{i}+\frac{1}{n} \sum_{j=1}^{n} z_{i, j} \\
& =r_{v_{i}} p_{v_{i}}+r_{v_{i}} p_{v_{i}+1} \frac{1}{\delta_{v_{i}}}\left(q_{v_{i}}-y_{i}\right)+2 y_{i}+\frac{1}{n} \sum_{j=1}^{n} z_{i, j} \\
& =1+\frac{1}{2 \delta_{v_{i}}}\left(q_{v_{i}}-y_{i}\right)+2 y_{i}+\frac{1}{n} \sum_{j=1}^{n} z_{i, j}
\end{aligned}
$$

Now we turn our interest to the term $\sum_{j=1}^{n} z_{i, j}$ and we derive the following lower bound using the indicator constraints (17). 


$$
\begin{aligned}
\sum_{j=1}^{n} z_{i, j} & =\sum_{j=1}^{v_{i}} z_{i, j}+\sum_{j=v_{i}+1}^{n} z_{i, j} \\
& \geq \sum_{j=1}^{v_{i}}\left(y_{i}-q_{j}\right)+\sum_{j=v_{i}+1}^{n}\left(q_{j}-y_{i}\right) \\
& =\sum_{j=1}^{v_{i}} y_{i}+\sum_{j=v_{i}+1}^{n}\left(-y_{i}\right)+\sum_{j=1}^{v_{i}}\left(-q_{j}\right)+\sum_{j=v_{i}+1}^{n} q_{j} \\
& =v_{i} y_{i}+\left(n-v_{i}\right)\left(-y_{i}\right)+\sum_{j=1}^{v_{i}}\left(-q_{j}\right)+\sum_{j=v_{i}+1}^{n} q_{j} \\
& =\left(2 v_{i}-n\right) y_{i}+\sum_{j=1}^{v_{i}}\left(-q_{j}\right)+\sum_{j=v_{i}+1}^{n} q_{j}
\end{aligned}
$$

Inserting (29) into (28) yields

$$
\begin{aligned}
T(i) & \geq 1+\frac{1}{2 \delta_{v_{i}}}\left(q_{v_{i}}-y_{i}\right)+2 y_{i}+\frac{1}{n}\left(\left(2 v_{i}-n\right) y_{i}+\sum_{j=1}^{v_{i}}\left(-q_{j}\right)+\sum_{j=v_{i}+1}^{n} q_{j}\right) \\
& =1+\frac{1}{2 \delta_{v_{i}}} q_{v_{i}}-\frac{1}{2 \delta_{v_{i}}} y_{i}+2 y_{i}+\frac{1}{n}\left(2 v_{i}-n\right) y_{i}+\frac{1}{n} \sum_{j=1}^{v_{i}}\left(-q_{j}\right)+\frac{1}{n} \sum_{j=v_{i}+1}^{n} q_{j}
\end{aligned}
$$

Let us check that the terms involving $y_{i}$ actually cancel out:

$$
\begin{aligned}
-\frac{1}{2 \delta_{v_{i}}} y_{i}+2 y_{i}+\frac{1}{n}\left(2 v_{i}-n\right) y_{i} & =\left(-\frac{n+2 v_{i}}{n}+\frac{2 n}{n}+\frac{2 v_{i}-n}{n}\right) \cdot y_{i} \\
& =\frac{-n-2 v_{i}+2 n+2 v_{i}-n}{n} \cdot y_{i} \\
& =0
\end{aligned}
$$

Inserting this fact into (30) yields

$$
T(i) \geq 1+\frac{1}{2 \delta_{v_{i}}} q_{v_{i}}+\frac{1}{n} \sum_{j=1}^{v_{i}}\left(-q_{j}\right)+\frac{1}{n} \sum_{j=v_{i}+1}^{n} q_{j}
$$

A few simple transformations bring us to the same terms as in the follower cost $C$ for our proposed solution; see (21). First, let us bring $\frac{1}{2 \delta_{v_{i}}} q_{v_{i}}$ into a more useful form:

$$
\frac{1}{2 \delta_{v_{i}}} q_{v_{i}}=\frac{n+2 v_{i}}{n} q_{v_{i}}=2 q_{v_{i}}+\frac{1}{n}\left(v_{i} q_{v_{i}}+\left(v_{i}-n\right) q_{v_{i}}\right)
$$


Combining (32) and (33) we get

$$
\begin{aligned}
T(i) & \geq 1+2 q_{v_{i}}+\frac{1}{n}\left(v_{i} q_{v_{i}}+\left(v_{i}-n\right) q_{v_{i}}\right)+\frac{1}{n} \sum_{j=1}^{v_{i}}\left(-q_{j}\right)+\frac{1}{n} \sum_{j=v_{i}+1}^{n} q_{j} \\
& =1+2 q_{v_{i}}+\frac{1}{n}\left(v_{i} q_{v_{i}}+\sum_{j=1}^{v_{i}}\left(-q_{j}\right)\right)+\frac{1}{n}\left(\left(v_{i}-n\right) q_{v_{i}}+\sum_{j=v_{i}+1}^{n} q_{j}\right) \\
& =1+2 q_{v_{i}}+\frac{1}{n} \sum_{j=1}^{v_{i}}\left(q_{v_{i}}-q_{j}\right)+\frac{1}{n} \sum_{j=v_{i}+1}^{n}\left(q_{j}-q_{v_{i}}\right) \\
& =1+2 q_{v_{i}}+\frac{1}{n} \sum_{j=1}^{v_{i}}\left|q_{v_{i}}-q_{j}\right|+\frac{1}{n} \sum_{j=v_{i}+1}^{n}\left|q_{j}-q_{v_{i}}\right| \\
& =1+2 q_{v_{i}}+\frac{1}{n} \sum_{j=1}^{n}\left|q_{v_{i}}-q_{j}\right|
\end{aligned}
$$

Thus, combining the parts $T(i)$ back into $T$ we obtain

$$
\begin{aligned}
T & =c_{0}+\sum_{i=1}^{k} T(i) \\
& \geq c_{0}+\sum_{i=1}^{k}\left(1+2 q_{v_{i}}+\frac{1}{n} \sum_{j=1}^{n}\left|q_{v_{i}}-q_{j}\right|\right) \\
& =c_{0}+k+\sum_{i=1}^{k} 2 q_{v_{i}}+\sum_{i=1}^{k} \sum_{j=1}^{n} \frac{1}{n}\left|q_{v_{i}}-q_{j}\right| \\
& =C .
\end{aligned}
$$

Thus, given the prices $d_{0}, \ldots, d_{k}$, the target function of the LP is lower bounded by $C$, the cost of our proposed solution. Thus, the solution is indeed of minimum follower cost and we already showed it to be feasible and to have a leader payoff of $k+c_{0}$.

(ii) Solution with high leader payoff implies existence of a $k$-clique. Assume now that there are leader prices $d_{0}, d_{1}, \ldots, d_{k}$ such that at least one optimal solution for the follower (i.e., an optimal solution to the LP) gives a leader payoff of at least $k+c_{0}$. We need to show that this implies the existence of a $k$-clique in the given instance $(G, k)$ of MULTICOLORED CLIQUE $(k)$.

Let $(w, x, y, z)$ an optimal solution for the follower, i.e., of minimum total cost taking into account the given leader prices, and among such solution achieves the maximum leader payoff. We, thus have $d^{T} x \geq k+c_{0}$. (Optimality of leader payoff will save us one argument later.) Our proof strategy is as follows:

1. Prove that $d_{i} x_{i} \leq 1$ for all $i \in[k]$, i.e., that the leader payoff per variable $x_{i}$ is at most 1 . This will imply that we have $d_{0} x_{0}=c_{0}$ and $d_{i} x_{i}=1$ for all $i \in[k]$. 
2. Prove that $x_{i} \in\left\{p_{1}, \ldots, p_{n}\right\}$. It can then be concluded that $\left(x_{i}, y_{i}\right) \in$ $\left\{\left(p_{1}, q_{1}\right), \ldots,\left(p_{n}, q_{n}\right)\right\}$. (This then also implies that all variables $w_{i, \ell}$ are equal to 0 by optimality, but we will not need it.)

3. At this point, using $d_{0} x_{0}=c_{0}$ yields $y_{0}=0$ and effectively makes all cliquetesting constraints active. The indicator variables $z_{i, j}$ work as intended, since $y_{i} \in\left\{q_{1}, \ldots, q_{n}\right\}$, and since the cost for increasing them beyond $\left|y_{i}-q_{j}\right|$ is larger than that for increasing $y_{0}$ to "fix" clique constraints. This yields the claimed $k$-clique.

Steps 1 and 2 are the main work, but fortunately they come down to similar indirect proofs that rule out bad cases by rather technical replacement arguments that would yield a solution with lower total cost. To facilitate this we give the replacement argument in the following technical claim before pursuing the above plan.

Claim 9 If $p_{s} \geq x_{i}>p_{s+1}$ and $d_{i} x_{i} \geq 1$ then $d_{i} \leq r_{s}$.

The proof of this claim is rather extensive and the reader is encouraged to skip ahead at first reading and check that the claim indeed leads to a reasonably fast conclusion of the main proof.

Proof of Claim 9 Assume for contradiction that $d_{i}>r_{s}$, and let $t \in\{s, \ldots, n\}$ with $r_{t}<d_{i} \leq r_{t+1}$. We will show that replacing $\left(x_{i}, y_{i}\right)$ by $\left(p_{t+1}, q_{t+1}\right)$, including further necessary updates to variables, yields a strictly cheaper follower solution, contradicting optimality of follower cost. (Recall that our assumed solution has minimum follower cost and, among such solutions, has maximum leader payoff.)

First, let us observe that we must have $y_{i}<q_{s+1}$ : Indeed, if this was not the case then $x_{i}>p_{s+1}$ and $y_{i} \geq q_{s+1}$. This, however, would contradict optimality of the follower cost since we can safely lower the value of $x_{i}$ to $p_{s+1}$ as the point $\left(x_{i}, y_{i}\right)=\left(p_{s+1}, q_{s+1}\right)$ fulfills all constraints of type (15) as well as $x_{i} \geq 0$ and $y_{i} \geq 0$ and having $y_{i}>p_{s+1}$ does not harm this. Since this would lower the follower cost, it cannot happen that $y_{i} \geq q_{s+1}$, as claimed. (This may be a good point to recall that the followers task/goal is only to get the minimum total cost, not to give a certain leader payoff; optimizing leader payoff is only a tiebreaker.)

Consider the variables $w_{i, \ell}$ : If $\ell \geq t+2$ then the cost of $w_{i, \ell}$ is $r_{\ell} \geq r_{t+2}>$ $r_{t+1}>d_{i}$. Thus, if $w_{i, \ell}>0$ then we could replace by $w_{i, \ell}^{\prime}=0$ and $x_{i}^{\prime}=x_{i}+w_{i, \ell}$, changing the cost by $\left(d_{i}-r_{\ell}\right) w_{i, \ell}<0$. Since $w_{i, \ell}$ only appears together with $x_{i}$ and with same coefficient, this would preserve feasibility. Since the replacement would contradict optimality of follower cost, it follows that $w_{i, \ell}=0$ for $\ell \geq t+2$. The same argument works also for $w_{i, t+1}$ if $d_{i}<r_{t+1}$. If, however, $d_{i}=r_{t+1}$ then we can appeal to optimality of the leader payoff: Indeed, if $d_{i}=r_{t+1}$ then making the analogous replacement would not affect the follower cost but it would increase the leader payoff, contradicting optimality of the latter among solutions with minimum follower cost. Thus, overall we get that

$$
w_{i, \ell}=0 \quad \text { for } \ell \geq t+1
$$

The situation is the opposite for $w_{i, t}$ : This variable has cost $r_{t}<d_{i}$ and replacing $w_{i, t}^{\prime}=w_{i, t}+\lambda$ and $x_{i}^{\prime}=x_{i}-\lambda$ for any $\lambda>0$ would change the total cost by 
$\lambda\left(r_{t}-d_{i}\right)<0$. This would lower the follower cost and contradict optimality of the follower cost. Thus, there is no $\lambda>0$ such that the replacement is feasible, implying that some constraint containing $x_{i}$ but not $w_{i, t}$ is already tight. Here it is crucial that $x_{i}$ and $w_{i, t}$ appear only positively and with same coefficient of 1 on the left hand side of $\geq$ constraints; see (15) and $x_{i} \geq 0$. Note that the later being tight would give $x_{i}=0$ and contradict $x_{i}>p_{s+1}$ thus it must be a constraint of type (15) for $j=u \geq t+1$ namely

$$
\begin{gathered}
x_{i}+\sum_{\ell=u}^{n} w_{i, \ell}+p_{u+1} \frac{1}{\delta_{u}} y_{i}=p_{u}+p_{u+1} \frac{1}{\delta_{u}} q_{u} \\
\Leftrightarrow x_{i}+p_{u+1} \frac{1}{\delta_{u}} y_{i}=p_{u}+p_{u+1} \frac{1}{\delta_{u}} q_{u},
\end{gathered}
$$

where the simplification uses the fact that $w_{i, \ell}=0$ for $\ell \geq t+1$, so in particular for $\ell \geq u \geq t+1$. For $j=t$ we get a similar simplification since only $w_{i, t}$ is not necessarily equal to 0 (and the constraint is not necessarily tight):

$$
\begin{aligned}
x_{i} & +\sum_{\ell=t}^{n} w_{i, \ell}+p_{t+1} \frac{1}{\delta_{t}} y_{i} \geq p_{t}+p_{t+1} \frac{1}{\delta_{t}} q_{t} \\
& \Leftrightarrow x_{i}+w_{i, t}+p_{t+1} \frac{1}{\delta_{t}} y_{i} \geq p_{t}+p_{t+1} \frac{1}{\delta_{t}} q_{t},
\end{aligned}
$$

Before using (37) and (38) for our replacement argument we would like to argue that in fact $u=t+1$, which reduces the number of different constants that we need to handle.

Assume for contradiction that $u \geq t+2$. It follows that $u-1 \geq t+1$ and hence that constraint $j=u-1$ of type (15) is also fulfilled with all $\bar{w}_{i, \ell}$ variables therein equal to 0 . It follows that we can discuss the constraints for $u$ and $u-1$ as constraints only on $x_{i}$ and $y_{i}$. First of all, (37) implies that $\left(x_{i}, y_{i}\right.$ must lie on the line defined by $\left(p_{u}, q_{u}\right)$ and $\left(p_{u+1}, q_{u+1}\right)$. Since the points $\left(p_{1}, q_{1}\right), \ldots,\left(p_{n+1}, q_{n+1}\right)$ are in strictly convex position, it follows that the points on the line that have $y_{i}$ coordinate strictly smaller than $q_{u}$ are not feasible for the preceding constraint, which is defined by $\left(p_{u-1}, q_{u-1}\right)$ and $\left(p_{u}, q_{u}\right)$. Thus, we get $y_{i} \geq q_{u}$, which is a contradiction since $q_{u} \geq q_{t+1} \geq q_{s+1}>y_{i}$. Thus, we must indeed have $u=t+1$, as claimed.

Let us recall the two constraints (37) and (38), with the latter updated to $u=t+1$, that we will use for the main replacement argument:

$$
\begin{gathered}
x_{i}+w_{i, t}+p_{t+1} \frac{1}{\delta_{t}} y_{i} \geq p_{t}+p_{t+1} \frac{1}{\delta_{t}} q_{t} \\
x_{i}+p_{t+2} \frac{1}{\delta_{t+1}} y_{i}=p_{t+1}+p_{t+2} \frac{1}{\delta_{t+1}} q_{t+1}
\end{gathered}
$$

We know that $y_{i}<q_{s+1}$ so, using $t \geq s$, we also have $y_{i}<q_{t+1}$. The remainder of the proof of the claim will focus on proving that updating to $\left(x_{i}, y_{i}\right)=\left(p_{s+1}, q_{s+1}\right)$ 
will strictly lower the follower cost. Thus, the assumption that $d_{i}>p_{s}$ must be wrong. There are two different cases for this, distinguished intuitively by whether $y_{i}$ is already close to $q_{t+1}$ or whether it is far (there is a clean cut-off). In the former case this yields a better lower bound for $d_{i}$ than $d_{i}>r_{t}$, which implies that the move saves enough cost due to lowering $x_{i}$ to $p_{s+1}$. In the latter case we cannot rule out that $d_{i}$ is arbitrarily close to $r_{t}$ (giving not enough savings) but we incur enough savings by carefully considering the cost change for indicator variables $z_{i, j}$.

Without further ado let us discuss the replacement step: The proposed replacement is $x_{i}^{\prime}=p_{s+1}, y_{i}^{\prime}=q_{s+1}$, setting all $w_{i, \ell}^{\prime}$ to 0 , and letting $z_{i, j}^{\prime}=\left|y_{i}^{\prime}-q_{j}\right|$. Finally, since this might lead to a violation of clique constraints for $z_{i, t+1}^{\prime}=0$, we increase $y_{0}$ sufficiently to compensate (and update $x_{0}$ to maintain $x_{0}+y_{0}=1$ ). Clearly, the replacement is feasible for (15) constraints since we use $\left(x_{i}, y_{i}\right)=\left(p_{s+1}, q_{s+1}\right)$. Similarly, constraints (17) are clearly fulfilled. Finally, the clique constraints (18) are fulfilled by increasing $y_{0}$ if necessary and $x_{0}+y_{0}=1$ is handled by updating $x_{0}^{\prime}=1-y_{0}^{\prime}$; we will postpone the discussion about the new values $x_{0}^{\prime}$ and $y_{0}^{\prime}$ till later, but clearly $x_{0}^{\prime}=0$ and $y_{0}^{\prime}=1$ always work.

It remains to consider the change in total cost that is incurred by this replacement; we denote this by $\Delta$. Using that $x_{0}^{\prime}-x_{0}=\left(1-y_{0}^{\prime}\right)-\left(1-y_{0}\right)=y_{0}-y_{0}^{\prime}=-\left(y_{0}^{\prime}-y_{0}\right)$, and plugging in known values for $x_{i}^{\prime}, y_{i}^{\prime}, w_{i, j}^{\prime}$, and some base prices we get

$$
\begin{aligned}
\Delta & =d_{0}\left(x_{0}^{\prime}-x_{0}\right)+c_{0}\left(y_{0}^{\prime}-y_{0}\right)+d_{i}\left(x_{i}^{\prime}-x_{i}\right)+c_{y}\left(y_{i}^{\prime}-y_{i}\right) \\
& +c_{z} \sum_{j=1}^{n}\left(z_{i, j}^{\prime}-z_{i, j}\right)+\sum_{j=1}^{n} r_{j}\left(w_{i, j}^{\prime}-w_{i, j}\right) \\
\leq & \left(c_{0}-d_{0}\right)\left(y_{0}^{\prime}-y_{0}\right)+d_{i}\left(p_{t+1}-x_{i}\right)+2\left(q_{t+1}-y_{i}\right) \\
& +\frac{1}{n} \sum_{j=1}^{n}\left(z_{i, j}^{\prime}-z_{i, j}\right)-\sum_{j=1}^{n} r_{j} w_{i, j} .
\end{aligned}
$$

We can also use the following simple lower bound

$$
\sum_{j=1}^{n} r_{j} w_{i, j} \geq r_{t} w_{i, t}
$$

whose application should make intuitive sense since $w_{i, t}$ appears in (38) and $w_{i, j}$ for $j \geq t+1$ are 0 . Plugging this into (40) yields the following upper bound for the cost.

$$
\begin{aligned}
\Delta \leq & \left(c_{0}-d_{0}\right)\left(y_{0}^{\prime}-y_{0}\right)+d_{i}\left(p_{t+1}-x_{i}\right)+2\left(q_{t+1}-y_{i}\right) \\
& +\frac{1}{n} \sum_{j=1}^{n}\left(z_{i, j}^{\prime}-z_{i, j}\right)-r_{t} w_{i, t}
\end{aligned}
$$

Considering that $d_{i}>r_{t}$, we have in (41) a contribution of $-r_{t}\left(x_{i}+w_{i, t}-p_{s+1}\right)$, which corresponds to cost savings incurred by the replacement. From (38) we can derive the 
following (where the first part replicates the argument for $\left(p_{t+1}, q_{t+1}\right)$ fulfilling this constraint):

$$
\begin{aligned}
x_{i}+w_{i, t}+p_{t+1} \frac{1}{\delta_{t}} y_{i} & \geq p_{t}+p_{t+1} \frac{1}{\delta_{t}} q_{t} \\
& =p_{t+1}+p_{t+1}+p_{t+1} \frac{1}{\delta_{t}} q_{t} \\
& =p_{t+1}+p_{t+1} \frac{1}{\delta_{t}}\left(\delta_{t}+q_{t}\right) \\
& =p_{t+1}+p_{t+1} \frac{1}{\delta_{t}} q_{t+1} \\
\Leftrightarrow \quad x_{i}+w_{i, t}-p_{t+1} & \geq p_{t+1} \frac{1}{\delta_{t}}\left(q_{t+1}-y_{i}\right) \\
\Leftrightarrow-r_{t}\left(x_{i}+w_{i, t}-p_{t+1}\right) & \leq-r_{t}\left(p_{t+1} \frac{1}{\delta_{t}}\left(q_{t+1}-y_{i}\right)\right) \\
& =-\frac{1}{2 \delta_{t}}\left(q_{t+1}-y_{i}\right)
\end{aligned}
$$

Plugging (42) into (41) we obtain

$$
\begin{aligned}
\Delta \leq & \left(c_{0}-d_{0}\right)\left(y_{0}^{\prime}-y_{0}\right)+\left(d_{i}-r_{t}\right)\left(p_{t+1}-x_{i}\right)+2\left(q_{t+1}-y_{i}\right) \\
& +\frac{1}{n} \sum_{j=1}^{n}\left(z_{i, j}^{\prime}-z_{i, j}\right)-\frac{1}{2 \delta_{t}}\left(q_{t+1}-y_{i}\right) \\
= & \left(c_{0}-d_{0}\right)\left(y_{0}^{\prime}-y_{0}\right)+\left(d_{i}-r_{t}\right)\left(p_{t+1}-x_{i}\right) \\
& +\left(2-\frac{1}{2 \delta_{t}}\right)\left(q_{t+1}-y_{i}\right)+\frac{1}{n} \sum_{j=1}^{n}\left(z_{i, j}^{\prime}-z_{i, j}\right) .
\end{aligned}
$$

Using

$$
2-\frac{1}{2 \delta_{t}}=2-\frac{2 n+4 t}{2 n}=\frac{4 n-(2 n+4 t)}{2 n}=\frac{n-2 t}{n}
$$

and plugging this into (43) we obtain

$$
\begin{aligned}
\Delta & \leq\left(c_{0}-d_{0}\right)\left(y_{0}^{\prime}-y_{0}\right)+\left(d_{i}-r_{t}\right)\left(p_{t+1}-x_{i}\right)+\left(\frac{n-2 t}{n}\right)\left(q_{t+1}-y_{i}\right) \\
& +\frac{1}{n} \sum_{j=1}^{n}\left(z_{i, j}^{\prime}-z_{i, j}\right) .
\end{aligned}
$$

As a next step we will need to branch into two cases, depending on the value of $\left(q_{t+1}-y_{i}\right)$, i.e., on the amount by which $y_{i}$ is changed; recall that $y_{i}<q_{t+1}$. Intuitively, the two cases behave as follows: 
1. $y_{i}$ is close to $q_{t+1}$ : The indicator variables $z_{i, j}$ change by an absolute value of $\left(q_{t+1}-y_{i}\right)$, with those for $q_{j}<y_{i}$ increasing and those for $q_{j}>y_{i}$ decreasing; this affects the fourth term in (44). Including the factor of $\frac{1}{n}$ we will see that this effectively cancels out with the third term in (44). We then use the tight constraint (39) to find that $x_{i}$ is "far" from $p_{t}$, which, using that $d_{i} x_{i} \geq 1$, implies that $d_{i}$ is "much larger" than $r_{t}$. This allows us to cancel out the first two terms in (44), as $\left(d_{i}-r_{t}\right)$ will be sufficiently large.

2. $y_{i}$ is far from $q_{t+1}$ : In this case it is possible that $d_{i}$ is arbitrarily close to $r_{t}$, which gives us a vanishing amount of savings from the term $\left(d_{i}-r_{t}\right)\left(p_{t+1}-x_{i}\right)$ in (44). Fortunately, if $y_{i}$ is sufficiently far from $q_{t+1}$ then the indicator variables, accounted for in the fourth term of (44), give additional savings.

We should note that the actual cutoff point chosen to distinguish the two cases is somewhat arbitrary. The first case gets progressively better as $y_{i}$ "gets closer to" $q_{t+1}$; doing the analysis in this way works roughly for $y_{i}$ equal to $q_{t-1}$ and larger. The second case gets better as $y_{i}$ gets smaller, and thus further away from $q_{t+1}$; savings for the indicator variables start at $y_{i}<q_{t}$. We choose a point roughly half way between $q_{t-1}$ and $q_{t}$ as the cutoff.

Case 1, $y_{i} \geq q_{t}-\frac{1}{2} \delta_{t}$ : We use the tight constraint for $j=t+1$, i.e., Eq. (39), to get an upper bound for $x_{i}$ :

$$
\begin{aligned}
x_{i}+p_{t+2} \frac{1}{\delta_{t+1}} y_{i} & =p_{t+1}+p_{t+2} \frac{1}{\delta_{t+1}} q_{t+1} \\
\Leftrightarrow \quad x_{i} & =p_{t+1}+p_{t+2} \frac{1}{\delta_{t+1}}\left(q_{t+1}-y_{i}\right) \\
& \leq p_{t+1}+p_{t+2} \frac{1}{\delta_{t+1}}\left(q_{t+1}-q_{t}+\frac{1}{2} \delta_{t}\right) \\
& =p_{t+1}+p_{t+2} \frac{1}{\delta_{t+1}}\left(\delta_{t}+\frac{1}{2} \delta_{t}\right) \\
& =p_{t+1}+p_{t+2} \frac{1}{\delta_{t+1}} \frac{3}{2} \delta_{t} \\
& =p_{t+1}+\frac{3}{2} \frac{\delta_{t}}{\delta_{t+1}} p_{t+2} \\
& =\frac{1}{2} p_{t}+\frac{3}{8} \frac{\delta_{t}}{\delta_{t+1}} p_{t} \\
& \leq \frac{15}{16} p_{t}
\end{aligned}
$$

In the last step we use

$$
\begin{aligned}
\frac{1}{2}+\frac{3}{8} \frac{\delta_{t}}{\delta_{t+1}} & =\frac{1}{2}+\frac{3}{8} \cdot \frac{n /(4 n+4 t)}{n /(4 n+4(t+1))}=\frac{1}{2}+\frac{3}{8} \cdot \frac{n+2 t+2}{n+2 t} \\
& =\frac{1}{2}+\frac{3}{8} \cdot\left(1+\frac{2}{n+2 t}\right)
\end{aligned}
$$




$$
\begin{aligned}
& \leq \frac{1}{2}+\frac{3}{8} \cdot\left(1+\frac{2}{n}\right)=\frac{1}{2}+\frac{3}{8} \cdot \frac{n+2}{n}=\frac{1}{2}+\frac{3 n+6}{8 n} \\
& =\frac{7 n+6}{8 n} \leq \frac{15}{16}
\end{aligned}
$$

where the final inequality uses $n \geq 12$ (rather than appealing to the fact that asymptotically we would get $\frac{7}{8}$ ). Note that $x_{i} \leq \frac{15}{16} p_{t}$ is a much stronger bound than what could be derived from the arrangement of (15) constraints; there we would need $y_{i}>q_{t}$ to get even just $x_{i}<p_{t}$. The point is that due to the presence of slack variables, in particular $w_{i, t}$, the point $\left(x_{i}, y_{i}\right)$ can lie "far" below the piecewise linear curve given by these constraints, as captured by the tight constraint (39) that we applied (including the knowledge about particular slack variables being zero).

Using $d_{i} x_{i} \geq 1$ it immediately follows that

$$
d_{i} \geq \frac{1}{x_{i}} \geq \frac{16}{15} \cdot \frac{1}{p_{t}}=\frac{16}{15} r_{t} .
$$

Now, we can return to Eq. (44); we begin by upper-bounding $\sum_{j=1}^{n}\left(z_{i, j}^{\prime}-z_{i, j}\right)$ :

$$
\sum_{j=1}^{n}\left(z_{i, j}^{\prime}-z_{i, j}\right)=\sum_{j=1}^{t}\left(z_{i, j}^{\prime}-z_{i, j}\right)+\sum_{j=t+1}^{n}\left(z_{i, j}^{\prime}-z_{i, j}\right)
$$

We plug in exact values for $z_{i, j}^{\prime}=\left|y_{i}^{\prime}-q_{j}\right|$ using $y_{i}^{\prime}=q_{t+1}$ and plug in lower bounds for $z_{i, j}$ obtained from constraints (17).

$$
\begin{aligned}
\sum_{j=1}^{n}\left(z_{i, j}^{\prime}-z_{i, j}\right) & \leq \sum_{j=1}^{t}\left(\left(q_{t+1}-q_{j}\right)-\left(y_{i}-q_{j}\right)\right)+\sum_{j=t+1}^{n}\left(\left(q_{j}-q_{t+1}\right)-\left(q_{j}-y_{i}\right)\right) \\
& =\sum_{j=1}^{t}\left(q_{t+1}-y_{i}\right)+\sum_{j=t+1}^{n}\left(y_{i}-q_{t+1}\right) \\
& =t \cdot\left(q_{t+1}-y_{i}\right)+(n-t) \cdot\left(y_{i}-q_{t+1}\right) \\
& =(2 t-n) \cdot\left(q_{t+1}-y_{i}\right)
\end{aligned}
$$

Now, plugging (47) into (44) we can upper bound $\Delta$ by

$$
\begin{aligned}
\Delta & \leq\left(c_{0}-d_{0}\right)\left(y_{0}^{\prime}-y_{0}\right)+\left(d_{i}-r_{t}\right)\left(p_{t+1}-x_{i}\right) \\
& +\left(\frac{n-2 t}{n}\right)\left(q_{t+1}-y_{i}\right)+\frac{1}{n} \sum_{j=1}^{n}\left(z_{i, j}^{\prime}-z_{i, j}\right)
\end{aligned}
$$




$$
\begin{aligned}
& \leq\left(c_{0}-d_{0}\right)\left(y_{0}^{\prime}-y_{0}\right)+\left(d_{i}-r_{t}\right)\left(p_{t+1}-x_{i}\right) \\
& +\left(\frac{n-2 t}{n}\right)\left(q_{t+1}-y_{i}\right)+\frac{1}{n}(2 t-n) \cdot\left(q_{t+1}-y_{i}\right) \\
& =\left(c_{0}-d_{0}\right)\left(y_{0}^{\prime}-y_{0}\right)+\left(d_{i}-r_{t}\right)\left(p_{t+1}-x_{i}\right) .
\end{aligned}
$$

Plugging in the lower bound (46) for $d_{i}$, and noting that $x_{i}>p_{s+1} \geq p_{t+1}$ implies $p_{t+1}-x_{i}<0$, we obtain

$$
\begin{aligned}
\Delta & \leq\left(c_{0}-d_{0}\right)\left(y_{0}^{\prime}-y_{0}\right)+\left(\frac{16}{15} r_{t}-r_{t}\right)\left(p_{t+1}-x_{i}\right) \\
& =\left(c_{0}-d_{0}\right)\left(y_{0}^{\prime}-y_{0}\right)+\frac{1}{15} r_{t}\left(p_{t+1}-x_{i}\right)
\end{aligned}
$$

Now, for the final step we need to upper bound the cost that is incurred in $\left(c_{0}-\right.$ $\left.d_{0}\right)\left(y_{0}^{\prime}-y_{0}\right)$ such that it is less than the absolute value of $\frac{1}{15} r_{t}\left(p_{t+1}-x_{i}\right)$. Note that the latter term vanishes for $x_{i}$ close to $p_{t+1}$ but, fortunately, this corresponds also to only a tiny movement of $y_{i}$, which in turn requires (at most) a tiny increase in $y_{0}$.

Let us consider the increase of $y_{0}$ that is necessary and sufficient when moving $y_{i}$ to $p_{t+1}$; this is captured by the value $y_{0}^{\prime}-y_{0}$. All new indicator values $z_{i, j}^{\prime}$ for $j \neq t+1$ are larger than $\varepsilon$ since $z_{i, j}^{\prime}$ was set to $\left|y_{i}^{\prime}-q_{j}\right|=\left|q_{t+1}-q_{j}\right|$ and the difference between different $q_{j}$ values is at least $\delta_{n} \geq \varepsilon$. Indicators for $i^{\prime} \in[k] \backslash\{i\}$ are not affected, so we only need to consider $z_{i, t+1}^{\prime}$ and $z_{i, t+1}$. We know that $z_{i, t+1}^{\prime}=\left|y_{i}^{\prime}-q_{t+1}\right|=0$, so all clique testing constraints (18) have their left hand side reduced by $z_{i, t+1}-z_{i, t+1}^{\prime}=$ $z_{i, t+1}$. Since they were satisfied before, it suffices to increase $y_{0}$ by $\frac{1}{\varepsilon} z_{i, t+1}$, noting the coefficient of $\varepsilon$ that $y_{0}$ has in such constraints. (We will of course not increase it above 1 , noting that $y_{0}=1$ yields $\varepsilon y_{0}=\varepsilon$ satisfying all clique-testing constraints.) Thus,

$$
\left(y_{0}^{\prime}-y_{0}\right) \leq \frac{1}{\varepsilon} z_{i, t+1} \text {. }
$$

We may assume that $z_{i, t+1}=\left|y_{i}-q_{t+1}\right|$ : Indeed, if it is larger then some cliquetesting constraint (18) must be tight, as constraints (17) are clearly not tight if $z_{i, t+1}>$ $\left|y_{i}-q_{t+1}\right|$. It follows that $z_{i, t+1}>0$ and hence that $y_{0}<1$. Then, however, we could decrease $z_{i, t+1}$ by some $\lambda>0$ and increase $y_{0}$ by $\frac{1}{\varepsilon} \lambda$, which overall changes the cost by $\left(c_{0} \cdot \frac{1}{\varepsilon}-c_{z}\right) \lambda<0$; a contradiction to optimality. (Here we assumed $c_{0}<\frac{\varepsilon}{n}$.) Thus, we can rewrite (50) to

$$
\left(y_{0}^{\prime}-y_{0}\right) \leq \frac{1}{\varepsilon} z_{i, t+1}=\frac{1}{\varepsilon}\left|y_{i}-q_{t+1}\right|=\frac{1}{\varepsilon}\left(q_{t+1}-y_{i}\right),
$$

as $y_{i}<q_{t+1}$. This in turn can be plugged into (49) to get

$$
\Delta \leq\left(c_{0}-d_{0}\right) \frac{1}{\varepsilon}\left(q_{t+1}-y_{i}\right)+\frac{1}{15} r_{t}\left(p_{t+1}-x_{i}\right)
$$


Now, we can apply constraint (39) again to express $\left(q_{t+1}-y_{i}\right)$ in terms of $\left(p_{t+1}-x_{i}\right)$ :

$$
\begin{array}{rlrl}
x_{i}+p_{t+2} \frac{1}{\delta_{t+1}} y_{i} & =p_{t+1}+p_{t+2} \frac{1}{\delta_{t+1}} q_{t+1} \\
\Leftrightarrow \quad p_{t+2} \frac{1}{\delta_{t+1}}\left(q_{t+1}-y_{i}\right) & =x_{i}-p_{t+1} \\
\Leftrightarrow & q_{t+1}-y_{i} & =\frac{1}{p_{t+2}} \delta_{t+1}\left(x_{i}-p_{t+1}\right) \\
\Leftrightarrow & q_{t+1}-y_{i} & =r_{t+2} \delta_{t+1}\left(x_{i}-p_{t+1}\right)
\end{array}
$$

Plugging (53) into (52) yields

$$
\Delta \leq\left(p_{t+1}-x_{i}\right) \cdot\left(\frac{1}{15} r_{t}-\left(c_{0}-d_{0}\right) \frac{1}{\varepsilon} r_{t+2} \delta_{t+1}\right) .
$$

Since $p_{t+1}-x_{i}<0$ and $\left(\frac{1}{15} r_{t}-\left(c_{0}-d_{0}\right) \frac{1}{\varepsilon} r_{t+2} \delta_{t+1}\right)>0$ as $c_{0}$ is sufficiently small, we get that

$$
\Delta<0
$$

which contradicts the assumed optimality of $(w, x, y, z)$ regarding total cost for the follower. A straightforward calculation shows that $c_{0}<\frac{\varepsilon}{30}$ suffices. It remains to consider the case that $y_{i}<q_{t}-\frac{1}{2} \delta_{t}$.

Case 2, $y_{i}<q_{t}-\frac{1}{2} \delta_{t}$ : As pointed out earlier, in this case we cannot (as far as we know) rule out that $d_{i}$ is arbitrarily close to $r_{t}$. Thus, we cannot follow the same arguments as in the previous case, as the term $\left(d_{i}-r_{t}\right)\left(p_{t+1}-x_{i}\right)$ in (48) could yield only a vanishing amount of savings that may not suffice to compensate for $\left(c_{0}-d_{0}\right)\left(y_{0}^{\prime}-y_{0}\right)$. Instead, we will revisit $\sum_{j=1}^{n}\left(z_{i, j}^{\prime}-z_{i, j}\right)$ and give a better upper bound using $y_{i}<q_{t}-\frac{1}{2} \delta_{t}$; we first replicate the analysis performed earlier, but leaving out the summand for $j=t$.

$$
\begin{aligned}
\sum_{j=1}^{n}\left(z_{i, j}^{\prime}-z_{i, j}\right)= & \sum_{j=1}^{t-1}\left(z_{i, j}^{\prime}-z_{i, j}\right)+\left(z_{i, t}^{\prime}-z_{i, t}\right)+\sum_{j=t+1}^{n}\left(z_{i, j}^{\prime}-z_{i, j}\right) \\
\leq & \sum_{j=1}^{t-1}\left(\left(q_{t+1}-q_{j}\right)-\left(y_{i}-q_{j}\right)\right)+\left(z_{i, t}^{\prime}-z_{i, t}\right) \\
& +\sum_{j=t+1}^{n}\left(\left(q_{j}-q_{t+1}\right)-\left(q_{j}-y_{i}\right)\right) \\
= & \sum_{j=1}^{t-1}\left(q_{t+1}-y_{i}\right)+\left(z_{i, t}^{\prime}-z_{i, t}\right)+\sum_{j=t+1}^{n}\left(y_{i}-q_{t+1}\right) \\
= & (t-1)\left(q_{t+1}-y_{i}\right)+\left(z_{i, t}^{\prime}-z_{i, t}\right)+(n-t)\left(y_{i}-q_{t+1}\right)
\end{aligned}
$$




$$
=(2 t-n-1)\left(q_{t+1}-y_{i}\right)+\left(z_{i, t}^{\prime}-z_{i, t}\right)
$$

For $\left(z_{i, t}^{\prime}-z_{i, t}\right)$ the point is that $y_{i}$ is initially smaller than $q_{t}$ by at least $\frac{1}{2} \delta_{t}$, causing $z_{i, t}$ to be at least $\frac{1}{2} \delta_{t}$. Intuitively, as $y_{i}$ is increased towards $y_{i}^{\prime}=q_{t+1}$ the value of $z_{i, t}$ first decreases to 0 (when reaching $q_{t}$ ) and then increases again; in particular, the difference between $z_{i, j}^{\prime}$ will be strictly less than $\left(q_{t+1}-y_{i}\right)$. Formally we get

$$
\begin{aligned}
\left(z_{i, t}^{\prime}-z_{i, t}\right) & \leq\left(q_{t+1}-q_{t}\right)-\left(q_{t}-y_{i}\right) \\
& =q_{t+1}-2 q_{t}+y_{i} \\
& <q_{t+1}-2 y_{i}-\delta_{t}+y_{i} \\
& =q_{t+1}-y_{i}-\delta_{t}
\end{aligned}
$$

using $-q_{t}<-y_{i}-\frac{1}{2} \delta_{t}$ to replace $-2 q_{t}$ in the second line. Plugging (56) into (55) we get

$$
\begin{aligned}
\sum_{j=1}^{n}\left(z_{i, j}^{\prime}-z_{i, j}\right) & <(2 t-n-1)\left(q_{t+1}-y_{i}\right)+q_{t+1}-y_{i}-\delta_{t} \\
& =(2 t-n)\left(q_{t+1}-y_{i}\right)-\delta_{t}
\end{aligned}
$$

Plugging (57) into (44) we get almost the same cancellation as for $y_{i} \geq q_{t}-\frac{1}{2} \delta_{t}$ except for getting extra savings of $-\delta_{t}$ :

$$
\begin{aligned}
\Delta \leq\left(c_{0}-d_{0}\right)\left(y_{0}^{\prime}-y_{0}\right)+\left(d_{i}-r_{t}\right)\left(p_{t+1}-x_{i}\right)+\left(\frac{n-2 t}{n}\right)\left(q_{t+1}-y_{i}\right) \\
+\frac{1}{n} \sum_{j=1}^{n}\left(z_{i, j}^{\prime}-z_{i, j}\right) \\
\quad<\left(c_{0}-d_{0}\right)\left(y_{0}^{\prime}-y_{0}\right)+\left(d_{i}-r_{t}\right)\left(p_{t+1}-x_{i}\right) \\
\quad+\left(\frac{n-2 t}{n}\right)\left(q_{t+1}-y_{i}\right)+\frac{1}{n}\left((2 t-n)\left(q_{t+1}-y_{i}\right)-\delta_{t}\right) \\
\quad=\left(c_{0}-d_{0}\right)\left(y_{0}^{\prime}-y_{0}\right)+\left(d_{i}-r_{t}\right)\left(p_{t+1}-x_{i}\right)-\frac{1}{n} \delta_{t}
\end{aligned}
$$

We are now almost done (with both the second case and the claim). We can upper bound $\left(c_{0}-d_{0}\right)\left(y_{0}^{\prime}-y_{0}\right)$ by $c_{0}$ since $d_{0} \geq 0, y_{0}^{\prime} \leq 1$, and $y_{0} \geq 0$. Since $c_{0}$ is sufficiently small, this is strictly less than $\frac{1}{n} \delta_{t}$. It suffices to choose $c_{0} \leq \frac{1}{7 n}$ here, since $\delta_{t} \geq \frac{1}{6}$. Similarly, we know that $\left(d_{i}-r_{t}\right)\left(p_{t+1}-x_{i}\right)<0$ since $d_{i}>r_{t}$ and $x_{i}>p_{t+1}$. Thus, plugging in these inequalities yields

$$
\Delta<0
$$


which, again, contradicts optimality of the solution $(w, x, y, z)$ regarding the follower cost. It follows that both cases for $y_{i}$ are impossible, which means that the initial assumption of $d_{i}>r_{s}$ must be wrong. This implies $d_{i} \leq r_{s}$ and proves the claim.

After this piece of work we now have a powerful claim to wield in order to handle the two main steps for reasoning about optimal follower solutions with leader payoff at least $k+c_{0}$. To recall, we know that if $d_{i} x_{i} \geq 1$ and $p_{s} \geq x_{i}>p_{s+1}$ then $d_{i} \leq r_{s}$.

1. Assume that for some $i \in[k]$ we have $d_{i} x_{i}>1$ and that $p_{s} \geq x_{i}>p_{s+1}$ for some $s \in[n]$. (The special cases of $x_{i}>p_{1}$ and $p_{n+1} \geq x_{i}$ will be handled later.) It follows from Claim 9 that $d_{i} \leq r_{s}$, giving a direct contradiction. Thus, we have $d_{i} x_{i} \leq 1$ for all $i \in[k]$.

If $x_{i}>p_{1}$ then the solution cannot be optimal: Indeed, we have $y_{i} \geq 0$ so lowering $x_{i}$ to $x_{i}^{\prime}=p_{1}$ will, by construction, fulfill all constraints (15) even if all variables $w_{i, \ell}$ are equal to 0 . This would incur a cost change of $d_{i}\left(x_{i}^{\prime}-x_{i}\right)<0$ and contradict optimality of follower cost.

If $p_{n+1} \geq x_{i}$ then $d_{i}>\frac{1}{x_{i}} \geq \frac{1}{p_{n+1}}=r_{n+1}>r_{n}$. If lowering $x_{i}$ by some $\lambda>0$ and increasing $w_{i, n}$ by the same amount would be feasible then this would change the total cost by $\left(r_{n}-d_{i}\right) \lambda<0$ since $w_{i, n}$ has cost $r_{n}$. It follows that there must be a constraint containing $x_{i}$ but not $w_{i, n}$ that is already tight (recalling that both occur only on the left hand side of $\geq$ constraints and always with coefficient 1 ). The only such constraint is $x_{i} \geq 0$, but this being tight means that $x_{i}=0$, contradicting the assumption that $d_{i} x_{i} \geq 1$. Thus, neither $x_{i}>p_{1}$ nor $x_{i} \leq p_{n}$ are possible if $d_{i} x_{i} \geq 1$.

Recall that we have $x_{0}+y_{0}=1$ with $\operatorname{cost} c_{0}$ for $y_{0}$ and leader cost $d_{0}$ (but no base cost) for $x_{0}$. Since only $y_{0}$ appears in other constraints, and only on the left hand side of $\geq$ constraints, increasing it while decreasing $x_{0}$ does not violate any constraints (that is, so long as $x_{0} \geq 0$ and $y_{0}=1-x_{0} \leq 1$ ). Thus, if $d_{0}>c_{0}$ then optimal follower solutions have $x_{0}=0$ and hence $d_{0} x_{0}=0$. Since we already know that $d_{i} x_{i} \leq 1$ for $i \in[k]$ it follows that $d_{0} x_{0} \geq c_{0}$. Since $x_{0} \leq 1$, due to $x_{0}+y_{0}=1$ and $y_{0} \geq 0$, it follows that

$$
d_{0} x_{0}=c_{0} \quad d_{i} x_{i}=1 \quad \text { for } i \in[k],
$$

including that $x_{0}=1$ and $y_{0}=0$ since otherwise the total leader profit would be strictly less than $k+c_{0}$. (We now have that it is exactly $k+c_{0}$.)

2. Assume now that $p_{s}>x_{i}>p_{s+1}$ for some $i \in[k]$, i.e., that some $x_{i}$ does not take one of the $n$ intended values $\left\{p_{1}, \ldots, p_{n}\right\}$. Using $d_{i} x_{i}=1$ we get $d_{i}=\frac{1}{x_{i}}>\frac{1}{p_{s}}=$ $r_{s}$. This, however, contradicts Claim 9 which, for $d_{i} x_{i} \geq 1$ and $p_{s} \geq x_{i}>p_{s+1}$ implies $d_{i} \leq r_{s}$. Thus, we must have $x_{i} \in\left\{p_{1}, \ldots, p_{n}\right\}$ for all $i \in[k]$.

Pick any $i \in[k]$ and let $s \in[n]$ with $x_{i}=p_{s}$ and $d_{i}=r_{s}$, and consider variables $w_{i, \ell}$ with $\ell \geq s$ : If $\ell>s$ then having $w_{i, \ell}>0$ would allow to decrease it by some $\lambda>0$ and increase $x_{i}$ by $\lambda$ without breaking feasibility, but changing the follower cost by $\left(d_{i}-r_{\ell}\right) \lambda<0$, as $w_{i, \ell}$ has cost $r_{\ell}>r_{s}=d_{i}$. Similarly, if $\ell=s$ then $w_{i, \ell}=0$ would allow the same modification; in this case, the follower cost would remain the same as $r_{\ell}=r_{s}=d_{i}$ but the leader payoff would 
increase, contradicting its optimality among solutions of minimum follower cost. This implies (by feasibility) that

$$
x_{i}+p_{s+1} \frac{1}{\delta_{s}} y_{i} \geq p_{s}+p_{s+1} \frac{1}{\delta_{s}} q_{s},
$$

by taking constraint (15) for $j=s$ and using that $w_{i, \ell}=0$ for $\ell \geq s$. Plugging in $x=p_{s}$ we directly get that $y_{i} \geq q_{s}$. If indeed $y_{i}>q_{s}$ then by construction of (15) constraints the point $\left(x_{i}, y_{i}\right)$ is strictly fulfilling all of them, and $x_{i}$ could be lowered by some $\lambda>0$ without breaking feasibility, but lowering the follower cost and causing a contradiction. Thus, $y_{i}=q_{s}$, as planned in the proof strategy.

3. We know now that for each $i \in[k]$ the solution fulfills $\left(x_{i}, y_{i}\right) \in\left\{\left(p_{1}, q_{1}\right), \ldots\right.$, $\left.\left(p_{n}, q_{n}\right)\right\}$ and $d_{i} x_{i}=1$. To check that the $y_{i}$ variables indeed correspond to a $k$-clique, let us recall the simple argument giving $z_{i, j}=\left|y_{i}-q_{j}\right|$ that we used when proving Claim 9: If $z_{i, j}>\left|y_{i}-q_{j}\right|$ then there is $\lambda>0$ such that lowering $z_{i, j}$ by $\lambda$ will preserve $z_{i, j} \geq\left|y_{i}-q_{j}\right|$ and lower the cost. The only constraints that could now be violated would be clique-testing constraints (18) that involve $z_{i, j}$. These however can be fixed by increasing $y_{0}^{\prime}$ by $\frac{1}{\varepsilon} \lambda$ (or possibly only to 1 ), which in total yields a decrease in cost, as $\frac{1}{\varepsilon} c_{0}<c_{z}$; a contradiction. It follows that $z_{i, j}=0$ if $y_{i}=q_{j}$.

Let $v_{1}, \ldots, v_{k} \in[n]$ such that $y_{i}=q_{v_{i}}$. We claim that $\left\{\left(1, v_{1}\right), \ldots,\left(k, v_{k}\right)\right\}$ is a $k$-clique in $G$. Consider any two vertices $\left(i, v_{i}\right)$ and $\left(j, v_{j}\right)$ for $i \neq j$. If $\left\{\left(i, v_{i}\right),\left(j, v_{j}\right)\right\}$ was not an edge of $G$ then there would be a corresponding cliquetesting constraint of type (18):

$$
z_{i, v_{i}}+z_{j, v_{j}}+\varepsilon y_{0} \geq \varepsilon
$$

By the above discussion we know that $z_{i, v_{i}}=z_{j, v_{j}}=0$ and we previously determined that $y_{0}=0$. This, however, would imply that the constraint is violated, contradicting feasibility. Thus, for all $i \neq j$, the vertices $\left(i, v_{i}\right)$ and $\left(j, v_{j}\right)$ are adjacent in $G$ and, accordingly $\left\{\left(1, v_{1}\right), \ldots,\left(k, v_{k}\right)\right\}$ is a $k$-clique in $G$ as claimed. This completes the correctness proof.

We have given a polynomial-time reduction from the W[1]-hard MULTICOLORED CLIQUE $(k)$ problem that only increases the parameter value by 1 . This implies W[1]hardness of LP- PRICING, completing the proof.

Theorem 4 is in sharp contrast to the combinatorial setting, where under mild assumptions one can see the problem to be fixed-parameter tractable. Here we assume that $X \subseteq\{0,1\}^{n}$ but put no further restriction on the follower's objective function $f: X \rightarrow \mathbb{R}$. In particular, this model covers the classical setting where each item has a fixed cost and if the follower buys a set $S$ of items, he has to pay the sum of the fixed costs of the elements of $S$, plus the leader's price of the bought elements.

Theorem 5 Assume that $X \subseteq\{0,1\}^{n}$, and that we are given a polynomial-time algorithm to compute an optimal solution of the follower for given leader prices $p \in \mathbb{R}^{k}$. Then the computation of optimal prices and optimal leader revenue is fixed-parameter 
tractable, with running time $\mathcal{O}\left(2^{k} n^{c}\right)$, when parameterized by the number of priceable items.

In the above statement we make the natural assumption that the input size is at least $n+\operatorname{size}(f)$, where size $(f)$ denotes the maximum length of the binary encoding of any value $f$ can take.

Proof We need the following proposition due to Briest et al. [10]. It says that if the leader wants to force the follower to pick a certain solution, she can compute suitable prices in polynomial time. We state their result in a slightly more general fashion than in the original paper. Indeed, Briest et al. were only concerned with the case when $f$ is additive ${ }^{4}$ but the proof did not make use of the additivity of $f$ at all. We refrain from restating the proof in order to avoid redundancy.

Proposition 2 (Briest et al. [10]) Given a vector $z \in X$, one can compute an optimal price vector $p$ such that $z$ is an optimal solution of the follower with respect to $p$, or decide that such a price vector does not exist, in polynomial time.

Our algorithm works as follows. For each vector $x \in\{0,1\}^{k}$ we compute a price vector $p_{x}$, if exists, such that

(a) there is some $y \in\{0,1\}^{n-k}$ such that the vector $(x, y)$ is an optimal solution of the follower with respect to the price vector $p_{x}$,

(b) subject to (a) the revenue $p_{x}^{T} x$ is maximum.

When this procedure is finished we choose the vector $\hat{x} \in\{0,1\}^{k}$ with maximum value of $p_{\hat{x}}^{T} \hat{x}$, and output the price vector $p_{\hat{x}}$. (Note that the algorithm does indeed output something since, at the very least, the vector $p_{0}$ exists, due to our global assumption that there is a follower's solution without support of the priceable variables. Here, $\mathbf{0}$ denotes the $k$-dimensional all-zeroes vector.) As the next claim shows, this price vector is the optimum solution.

Claim 10 The output $p_{\hat{x}}$ is an optimal price vector for the leader.

Proof To this end, let $r^{*}$ be the maximum revenue achievable by the leader and let $p^{*}$ be the corresponding price vector. Moreover, let $\left(x^{*}, y^{*}\right)$ be an optimal solution of the follower under the price vector $p^{*}$ with $p^{* T} x^{*}=r^{*}$. By definition, the vector $p_{x^{*}}$ is computed in the course of our algorithm and, by property (b), $p_{x^{*}}{ }^{T} x^{*} \geq p^{* T} x^{*}$. Since $p_{\hat{x}}$ was the output price vector we have $p_{\hat{x}}^{T} x \geq p_{x^{*}}{ }^{T} x^{*}$, and so

$$
p_{\hat{x}}^{T} \hat{x} \geq p_{x^{*}}^{T} x^{*} \geq p^{* T} x^{*}=r^{*},
$$

as desired.

In the remainder of the proof we show how to compute $p_{x}$ for a fixed candidate vector $x \in\{0,1\}^{k}$. First we aim to find a vector $y_{x} \in\{0,1\}^{n-k}$ such that $\left(x, y_{x}\right) \in X$

\footnotetext{
${ }^{4}$ Recall that $f$ is additive if and only if for each $x \in X$ the following holds: if $x=\sum_{i=1}^{n} \lambda_{i} \cdot e_{i}$, where $e_{i}$ denotes the $i$-th unit vector, then $\sum_{i=1}^{n} \lambda_{i} \cdot f\left(e_{i}\right)$.
} 
and, subject to this, $f\left(x, y_{x}\right)$ is minimum. Note that possibly such a vector $y_{x}$ does not exist. To find a vector $y_{x}$, or decide that none exists, we define a price vector $p$ by setting

$$
p_{i}= \begin{cases}-M & \text { if } x_{i}=1 \\ M & \text { if } x_{i}=0\end{cases}
$$

for all $i \in[k]$. Here, $M$ is a number that is large enough to ensure that

$$
\text { for all }\left(x^{\prime}, y^{\prime}\right),\left(x^{\prime \prime}, y^{\prime \prime}\right) \in X \text {, it holds that } f\left(x^{\prime}, y^{\prime}\right)-f\left(x^{\prime \prime}, y^{\prime \prime}\right)<M \text {. }
$$

As both values $\left|f\left(x^{\prime}, y^{\prime}\right)\right|$ and $\left|f\left(x^{\prime \prime}, y^{\prime \prime}\right)\right|$ are bounded by $2^{\text {size }(f)}$, we may simply put $M=2^{\operatorname{size}(f)+1}+1$.

Now we compute an optimal solution of the follower with respect to the price vector $p$, say $\left(x^{*}, y^{*}\right)$, using the assumed polynomial-time algorithm.

Claim 11 If for some $y \in\{0,1\}^{n-k}$ it holds that $(x, y) \in X$, then $x^{*}=x$.

Proof To see Claim 11, suppose it is false. So, there is some vector $y \in\{0,1\}^{n-k}$ with $(x, y) \in X$, but $x^{*} \neq x$. By the choice of $p$, we know that

$$
p^{T} x^{*} \geq p^{T} x+M,
$$

and thus

$$
p^{T} x^{*}+f\left(x^{*}, y^{*}\right) \stackrel{(60)}{\geq} p^{T} x+M+f\left(x^{*}, y^{*}\right) \stackrel{(59)}{>} p^{T} x+f(x, y),
$$

a contradiction to the optimality of $\left(x^{*}, y^{*}\right)$. This proves Claim 11.

If $x^{*} \neq x$, Claim 11 implies that there is no price vector satisfying (a). Thus, we may safely abort the process and go over to the next candidate vector $x$. Otherwise if $x^{*}=x$, we put $y_{x}=y^{*}$.

Claim $12 f\left(x, y_{x}\right) \leq f(x, y)$ holds for all $y \in\{0,1\}^{n-k}$ with $(x, y) \in X$.

Proof If Claim 12 was wrong, $\left(x^{*}, y^{*}\right)$ would not have been the optimal solution of the follower under the price vector $p$, which is a contradiction. Hence, Claim 12 is valid.

Next, we use Proposition 2 to compute a price vector $p_{x}$ such that the vector $\left(x, y_{x}\right)$ is optimal for the follower and, subject to that, $p_{x}{ }^{T} x$ is maximum. Note that this price vector does exist since, e.g., the vector $p$ is a feasible price vector. By construction, $p_{x}$ has the property (a). So far, we only know that $p_{x}{ }^{T} x$ is maximum subject to the condition that under the price vector $p_{x}$ the vector $\left(x, y_{x}\right)$ is an optimal solution of the follower.

Claim 13 Subject to $(a)$, the revenue $p_{x}^{T} x$ is maximum. 
Proof To this end, let $p^{\prime}$ be any price vector for the leader such that (a) holds. That is, there is an optimal solution $\left(x, y^{\prime}\right)$ of the follower for some suitable $y^{\prime}$ under the price vector $p^{\prime}$.

Recall that, by Claim $12, f\left(x, y_{x}\right) \leq f\left(x, y^{\prime}\right)$, and so

$$
p^{\prime T} x+f\left(x, y_{x}\right) \leq p^{\prime T} x+f\left(x, y^{\prime}\right)
$$

This means that $\left(x, y_{x}\right)$ is also an optimal solution of the follower under the price vector $p^{\prime}$. Hence, due to the choice of $p_{x}$, we know that ${p^{\prime}}^{T} x \leq p_{x}^{T} x$. This implies (b) and the correctness of the algorithm.

As the running time of the whole algorithm is $O\left(2^{k} \cdot(n+\operatorname{size}(f))^{O(1)}\right)$, the proof is complete.

\section{Conclusion and future work}

The basis for the first part of this paper were the results of Briest et al. [10] and Balcan et al. [3] who gave a lower bound on the optimal revenue in Stackelberg Network Pricing Games. We proved that this bound carries over to a much more general setting, where, basically, the follower minimizes a continuous function over a compact set of points. This model captures important settings that are not covered by the classical combinatorial model. For example, the case when the follower is minimizing a linear program, e.g., a minimum cost flow problem.

The proven lower bound also holds if a single-price strategy is applied, and it is tight up to a factor of $(1+o(1))$. Moreover, we used this tightness example to show that it is strongly NP-hard to decide whether the revenue of an optimal pricing exceeds the lower bound by an arbitrarily small linear factor.

In the second part of the paper we studied the parameterized complexity of the revenue maximization problem. It turned out that in the combinatorial setting (i.e., when the follower only has 0/1-valued solutions) there is an elegant FPT algorithm. Once we leave this regime, however, things become more difficult. Indeed, if the follower has an optimization problem in the form of a linear program, the revenue maximization problem becomes W[1]-hard and is thus most likely not FPT.

Several central questions remain. Most importantly, one should consider multiplefollower scenarios. An intriguing model is when the particular resources have a limited supply. In the combinatorial setting, a limited supply means that every item to be sold is available only a limited number of times. Now the followers come one by one, in a certain order, and buy according to their preferences and the prices set by the leader. Balcan et al. [3] prove a tight lower bound on the revenue obtained by the single-price strategy. In the non-combinatorial model, the limited supply might be translated to a constraint of the form $(x, y) \leq s$, where $s \in \mathbb{R}_{\geq 0}^{n}$ is a fixed vector that is added to the usual constraint $(x, y) \in X$ in the optimization problem of the followers.

Acknowledgements Open Access funding provided by Projekt DEAL. 
Open Access This article is licensed under a Creative Commons Attribution 4.0 International License, which permits use, sharing, adaptation, distribution and reproduction in any medium or format, as long as you give appropriate credit to the original author(s) and the source, provide a link to the Creative Commons licence, and indicate if changes were made. The images or other third party material in this article are included in the article's Creative Commons licence, unless indicated otherwise in a credit line to the material. If material is not included in the article's Creative Commons licence and your intended use is not permitted by statutory regulation or exceeds the permitted use, you will need to obtain permission directly from the copyright holder. To view a copy of this licence, visit http://creativecommons.org/licenses/by/4.0/.

\section{References}

1. Aggarwal, G., Feder, T., Kenthapadi, K., Motwani, R., Panigrahy, R., Thomas, D., Zhu, A.: An: approximation algorithms for k-anonymity. J. Privacy Technol. (JOPT) (2005)

2. Baïou, M., Barahona, F.: Stackelberg bipartite vertex cover and the preflow algorithm. Algorithmica 74, 1174-1183 (2016)

3. Balcan, M.-F., Blum, A., Mansour, Y.: Item pricing for revenue maximization. In: Proceedings 9th ACM Conference on Electronic Commerce (EC-2008), Chicago, IL, USA, June 8-12, 2008, pp 50-59 (2008)

4. Berbeglia, G., Joret, G.: Assortment optimisation under a general discrete choice model: a tight analysis of revenue-ordered assortments. (2016) arXiv preprint arXiv:1606.01371

5. Bilò, D., Gualà, L., Leucci, S., Proietti, G.: Specializations and generalizations of the Stackelberg minimum spanning tree game. Theor. Comput. Sci. 562, 643-657 (2015)

6. Bilò, D., Gualà, L., Proietti, G., Widmayer, P.: Computational aspects of a 2-player Stackelberg shortest paths tree game. In: Internet and Network Economics, pp. 251-262. Springer (2008)

7. Borwein, J., Borwein, P.: Pi and the AGM: A Study in Analytic Number Theory and Computational Complexity. Wiley, Hoboken (1987)

8. Briest, P., Chalermsook, P., Khanna, S., Laekhanukit, B., Nanongkai, D.: Improved hardness of approximation for stackelberg shortest-path pricing. In: Internet and Network Economics, pp. 444-454. Springer (2010)

9. Briest, P., Gualà, L., Hoefer, M., Ventre, C.: On stackelberg pricing with computationally bounded customers. Networks 60(1), 31-44 (2012)

10. Briest, P., Hoefer, M., Krysta, P.: Stackelberg network Pricing Games. Algorithmica 62(3-4), 733-753 (2012)

11. Cabello, S.: Stackelberg shortest path tree game. Revisited (2012). arXiv preprint arXiv:1207.2317

12. Cardinal, J., Demaine, E.D., Fiorini, S., Joret, G., Langerman, S., Newman, I., Weimann, O.: The Stackelberg minimum spanning tree game. Algorithmica 59, 129-144 (2011)

13. Cardinal, J., Demaine, E.D., Fiorini, S., Joret, G., Newman, I., Weimann, O.: The Stackelberg minimum spanning tree game on planar and bounded-treewidth graphs. J. Comb. Optim. 25(1), 19-46 (2013)

14. Chakraborty, T., Huang, Z., Khanna, S.: Dynamic and non-uniform pricing strategies for revenue maximization. SIAM J. Comput. 42(6), 2424-2451 (2013)

15. Guruswami, V., Hartline, J.D., Karlin, A.R., Kempe, D., Kenyon, C., McSherry, F.: On profitmaximizing envy-free pricing. In: Proceedings of the Sixteenth Annual ACM-SIAM Symposium on Discrete Algorithms (SODA 2005), Vancouver, British Columbia, Canada, January 23-25, 2005, pp. 1164-1173 (2005)

16. Lenstra Jr., H.W.: Integer programming with a fixed number of variables. Math. Oper. Res. 8(4), 538-548 (1983)

17. Hagood, J.W., Thomson, B.S.: Recovering a function from a dini derivative. Am. Math. Mon. 113, 34-46 (2006)

18. Impagliazzo, R., Paturi, R., Zane, F.: Which problems have strongly exponential complexity? J. Comput. Syst. Sci. 63(4), 512-530 (2001)

19. Joret, G.: Stackelberg network pricing is hard to approximate. Networks 57(2), 117-120 (2011)

20. Labbé, M., Marcotte, P., Savard, G.: A Bilevel model of taxation and its application to optimal highway pricing. Manag. Sci. 44, 1608-1622 (1998)

21. Roche, S., Savard, G., Marcotte, P.: An approximation algorithm for Stackelberg network pricing. Networks 46, 57-67 (2005) 
22. van Hoesel, S.: An overview of Stackelberg pricing in networks. Eur. J. Oper. Res. 189, 1393-1402 (2008)

23. von Stackelberg, H.: Marktform und Gleichgewicht. Springer, Berlin (1934)

Publisher's Note Springer Nature remains neutral with regard to jurisdictional claims in published maps and institutional affiliations.

\section{Affiliations}

\section{Toni Böhnlein ${ }^{1}$. Stefan Kratsch ${ }^{2}$. Oliver Schaudt ${ }^{3}$}

$凶$ Toni Böhnlein

boehnlein@zpr.uni-koeln.de

Stefan Kratsch

kratsch@cs.uni-bonn.de

Oliver Schaudt

schaudt@mathc.rwth-aachen.de

1 Institut für Informatik, Universität zu Köln, Weyertal 80, 50931 Cologne, Germany

2 Institut für Informatik, Universität Bonn, Friedrich-Ebert-Allee 144, 53113 Bonn, Germany

3 Institut für Mathematik, RWTH Aachen University, Pontdriesch 10, 52062 Aachen, Germany 